\title{
Bifurcation analysis and integrability in the segmented disc dynamo with mechanical friction
}

\author{
Yebei Liu' ${ }^{1}$, Junze Li ${ }^{1}$, Zhouchao Wei ${ }^{1,2^{*}}$ and Irene Moroz ${ }^{3}$
}

"Correspondence:

weizhouchao@163.com

'School of Mathematics and

Physics, China University of

Geosciences, Wuhan, P.R. China

${ }^{2}$ Guangxi Colleges and Universities

Key Laboratory of Complex System

Optimization and Big Data

Processing, Yulin Normal University, Yulin, P.R. China

Full list of author information is

available at the end of the article

\begin{abstract}
Using center manifold theory, we investigate complex dynamics in a segmented disc dynamo with mechanical friction by making a determination of equilibrium states and analyzing local stability. We find that the system undergoes Hopf bifurcation for a key parameter. Normal form theory produces the formulae for determining a superor sub-critical Hopf bifurcation; the stability of the bifurcating limit cycle is also determined. In addition, integrability of the system is studied carefully.
\end{abstract}

Keywords: Segmented disc dynamo; Center manifold theory; Bifurcation; Chaos; Normal form theory; Integrability

\section{Introduction}

Since the first chaotic attractor in numerical experiments was discovered by E.N. Lorenz in the 1960s, chaos has achieved tremendous and far-reaching growth in many fields. Based on the existing chaotic systems, more researchers have focused on not only identifying new and interesting nonlinear systems, but also on enhancing complex dynamics and topological structure [1].

Model dynamos have been investigated over the past few decades to help to explicate how the magnetic field is reversed and generated in astrophysical bodies [2-8]. The selfexciting disc dynamo has been proposed as a simple prototype capable of dynamo action, which is deemed to be similar to the mechanism in both the convective envelope of the Sun and in the inner core of the Earth. Realizing that the analysis of the simplest disc dynamo was inconsistent, Moffatt proposed a segmented disc dynamo, where the current, related to radial diffusion of the magnetic field, could be included simply [5]. The frictionless dynamo is studied in the reference [4, 7]. In this paper we take into account mechanical friction, which can be described by the equations $[4,7]$ :

$$
\left\{\begin{array}{l}
\dot{x}=r(y-x)=P(x, y, z), \\
\dot{y}=x z+m x-(1+m) y=Q(x, y, z), \\
\dot{z}=g\left(1-(1+m) x y+m x^{2}\right)-f z=R(x, y, z),
\end{array}\right.
$$

(c) The Author(s) 2018. This article is distributed under the terms of the Creative Commons Attribution 4.0 International License (http://creativecommons.org/licenses/by/4.0/), which permits unrestricted use, distribution, and reproduction in any medium, provided you give appropriate credit to the original author(s) and the source, provide a link to the Creative Commons license, and indicate if changes were made. 
where $x(t)$ is the magnetic fluxes owing to radial and $y(t)$ denotes azimuthal currents, $z(t)$ measures the disc's angular velocity; $g$ represents the applied torque, $f$ is on behalf of the mechanical friction. Here $m, r$ are positive dimensionless parameters.

Based on the thought $[9,10]$ and no mechanical frictions, hidden chaotic solutions have been found, depending on the parameters of the problem [11]. In the case where friction is neglected $(f=0)$, the system will be structurally unstable and so not physically realistic [12]. However, Hide showed that it is 'unwarranted' for neglecting the mechanical friction in considering the two-disc dynamo [13]. A suitable choice of bifurcation parameter shows that Hopf bifurcations occur in equations (1.1). We will derive equations for deciding the direction of the bifurcating limit cycles and determine their linear stability by applying the theories of center manifolds and normal forms [14-16]. In the theory of polynomial differential equations, Darboux and Liouvillian integrals act a crucial role. Here we only study the Darboux integral of equations (1.1) by applying the theory of algebraic invariant surfaces, exponential factors, and Darboux polynomials (see [17-28]). We make further efforts to learn the behavior and geometry structure of equations (1.1) by studying their integrability. It turns out that there are no Darboux polynomials with cofactors being nonzero. And they have neither polynomial first integrals nor exponential factors. Furthermore, their first integrals of Darboux type do not exist either.

The paper is organized as follows. Section 2 investigates the dynamical behaviors of the segmented disc dynamo. In Sect. 3, normal form theory determines the super- or subcriticality of Hopf bifurcations and the stability of the bifurcating limit cycles. Section 4 considers integrability of system (1.1). And Sect. 5 contains our conclusions.

\section{Equilibria and local stability of system (1.1)}

Equations (1.1) are invariant under rotational symmetry about the $z$-axis, so that $(x, y, z) \rightarrow$ $(-x,-y, z)$. The divergence of the flow associated with (1.1) is $\nabla \cdot V=-(m+r+f+1)$. The system is therefore dissipative.

Central manifold theory gives Theorem 2.1.

Theorem 2.1 For $r>0, m>0, g>0$, and $f>0$, one can get the following four conclusions.

(i) If $f=g$, (1.1) has only one non-hyperbolic equilibrium: $P^{*}=(0,0,1)$; $P^{*}$ is asymptotically stable.

(ii) If $f<g,(1.1)$ has three equilibria $P^{+}=\left(\sqrt{1-\frac{f}{g}}, \sqrt{1-\frac{f}{g}}, 1\right), P^{-}=\left(-\sqrt{1-\frac{f}{g}},-\sqrt{1-\frac{f}{g}}\right.$, $1)$, and $P^{0}=\left(0,0, \frac{g}{f}\right) \cdot P^{0}$ is unstable, while $P^{ \pm}$are both asymptotically stable under the following criteria:

$$
\frac{2 r(g-f)}{r+f+m+1}<g(m+1)+r f
$$

(iii) If $f>g$, (1.1) has only one equilibrium $P^{0}=\left(0,0, \frac{g}{f}\right)$, and $P^{0}$ is asymptotically stable when

$$
(r f-r g)<(f+m+r+1)\left(r-\frac{r g}{f}+f+m f+r f\right) .
$$


Proof For case (i) with $f=g$, the change of variable $x_{1}=x, y_{1}=y, z_{1}=z-1$ gives

$$
\left\{\begin{array}{l}
\dot{x}_{1}=r\left(y_{1}-x_{1}\right), \\
\dot{y}_{1}=x_{1} z_{1}+(m+1) x_{1}-(1+m) y_{1}, \\
\dot{z}_{1}=g\left(-(1+m) x_{1} y_{1}+m x_{1}^{2}\right)-g z_{1} .
\end{array}\right.
$$

Hence, $E_{0}=(0,0,0)$ will be the only one equilibrium and its characteristic equation is

$$
\lambda^{3}+(g+m+r+1) \lambda^{2}+(r g+m g+g) \lambda=0 .
$$

The roots of equation (2.2) are $\lambda_{1}=0, \lambda_{2}=-g$, and $\lambda_{3}=-(1+m+r)$ with corresponding eigenvectors $\alpha_{1}=(1,1,0)^{T}, \alpha_{2}=(0,0,1)^{T}$, and $\alpha_{3}=\left(-\frac{r}{r+m}, 1,0\right)^{T}$, respectively, where $T_{1}$ denotes transpose.

Let

$$
\left(\begin{array}{l}
x_{1} \\
y_{1} \\
z_{1}
\end{array}\right)=T_{1}\left(\begin{array}{l}
u \\
v \\
w
\end{array}\right)
$$

where

$$
T_{1}=\left(\begin{array}{ccc}
1 & 0 & -\frac{r}{r+m} \\
1 & 0 & 1 \\
0 & 1 & 0
\end{array}\right)
$$

then system (2.1) becomes

$$
\left\{\begin{array}{l}
\dot{u}=\frac{r u v}{1+m+r}-\frac{r^{2} v w}{(1+m)(1+m+r)}, \\
\dot{v}=-g v-g u^{2}+g+g\left(-1-m+r-\frac{2 m r}{1+m}\right) u w+g\left(r+\frac{m r^{2}}{(1+m)^{2}}\right) w^{2}, \\
\dot{w}=-(1+m+r) w+\frac{1+m}{1+m+r} u v-\frac{r v w}{1+m+r} .
\end{array}\right.
$$

The linear stability of $E_{0}=(0,0,0)$ can be found from a first-order ordinary differential equation on a center manifold for the variable $u$ as follows:

$$
W^{c}(0)=\left\{u, v, w \in R \mid v=h_{1}(u), w=h_{2}(u), h_{j}(0)=0, D h_{j}(0)=0, j=1,2\right\}
$$

for $u$ sufficiently small.

Writing

$$
\begin{aligned}
& v=h_{1}(u)=a_{2} u^{2}+a_{3} u^{3}+O\left(u^{4}\right), \\
& w=h_{2}(u)=b_{2} u^{2}+b_{3} u^{3}+O\left(u^{4}\right)
\end{aligned}
$$

gives

$$
\mathscr{N}(h(x))=D_{x} h(x) A x+D_{x} h(x) f(x, h(x))-B h(x)-g(x, h(x))=0,
$$


where

$$
x=u, \quad y=(v, w), \quad h=\left(h_{1}, h_{2}\right), \quad A=0,
$$

and

$$
\begin{aligned}
& B=\left(\begin{array}{cc}
-g & 0 \\
0 & -(1+m+r)
\end{array}\right), \\
& f(x, y)=\frac{r v(u+m u-r w)}{(1+m)(1+m+r)} \\
& g(x, y)=\left(\begin{array}{c}
-\frac{\left.f(u+m u-r w)\left((1+m) u+\left(1+m^{2}+m(2+r)\right) w\right)\right)}{(1+m)^{2}} \\
\frac{v(u+m u-r w)}{1+m+r}
\end{array}\right) .
\end{aligned}
$$

Collecting the same terms and assigning the coefficients values of zero, we can get

$$
\begin{aligned}
u^{2}: & -g-g a_{2}=0 \quad \Rightarrow \quad a_{2}=-1, \\
& -(1+m+r) b_{2}=0 \quad \Rightarrow \quad b_{2}=0, \\
u^{3}: & -f a_{3}-\frac{f\left(1+m^{2}-r+m(2+r)\right) b_{2}}{1+m}=0 \quad \Rightarrow \quad a_{3}=0, \\
& \frac{(1+m) a_{2}}{(1+m+r)}-(1+m+r) b_{3}=0 \quad \Rightarrow \quad b_{3}=-\frac{1+m}{(1+m+r)^{2}} .
\end{aligned}
$$

Equations (2.4) and (2.6) give

$$
\begin{aligned}
& h_{1}(u)=-u^{2}+\cdots, \\
& h_{2}(u)=-\frac{1+m}{(1+m+r)^{2}} u^{3}+\cdots,
\end{aligned}
$$

which can approximate center manifold $W^{c}(0)$. Finally, substituting (2.7) into (2.3), the vector field restricted to the center manifold is given by

$$
\dot{u}=-\frac{r u^{3}}{1+m+r}-\frac{r^{2} u^{5}}{(1+m+r)^{3}}+\cdots .
$$

It follows from (2.8) that $u \rightarrow 0$ for $t \rightarrow+\infty$ and so $E_{0}$ is asymptotically stable. Therefore $P^{*}$ is also asymptotically stable.

For case (ii) with $f<g$, system (1.1) has three equilibria $P^{ \pm}\left( \pm \sqrt{1-\frac{f}{g}}, \pm \sqrt{1-\frac{f}{g}}, 1\right)$ and $P^{0}\left(0,0, \frac{g}{f}\right)$. The characteristic equations for both $P^{+}$and $P^{-}$are identical:

$$
\lambda^{3}+(1+m+r+f) \lambda^{2}+(g+r f+m g) \lambda+2 g r-2 r f=0 .
$$

Because of the symmetry of the system, it is enough if we just discuss $P^{+}$here.

According to the Routh-Hurwitz criterion, $P^{+}$and $P^{-}$are both asymptotically stable provided

$$
\frac{2 r(g-f)}{r+f+m+1}<g(m+1)+r f .
$$


The characteristic equation for $P^{0}$ is

$$
\lambda^{3}+(1+m+r+f) \lambda^{2}+\left(r+r f+m f+f-\frac{r g}{f}\right) \lambda+r f-r g=0
$$

It is straightforward to show that $P^{0}$ is unstable.

For case (iii) with $f>g$, system (1.1) has only one equilibrium $P^{0}=\left(0,0, \frac{g}{f}\right)$, which is asymptotically stable when

$$
(r f-r g)<(f+m+r+1)\left(r-\frac{r g}{f}+f+m f+r f\right) .
$$

\section{Hopf bifurcations in system (1.1)}

Due to the symmetry of the system, the equilibrium points $P^{+}$and $P^{-}$have the same bifurcation properties, so we confine our focus to $P^{+}$. Theorems 3.1 and 3.2 can be proved.

Theorem 3.1 When $0<m<1, r>\frac{(m+1)(m+f+1)}{1-m}$, and $g>f, P^{+}$, given in Theorem 2.1, undergoes a Hopf bifurcation when

$$
g=g_{0}=\frac{2 r+r(r+f+m+1)}{2 r-(m+1)(r+f+m+1)} f
$$

Proof When $P^{+}$undergoes a Hopf bifurcation, (2.9) has a conjugate complex eigenvalue, and the real part is zero. Assume $\lambda= \pm i \omega$.

When $\lambda=i \omega$, (2.9) becomes

$$
-2 f r+2 g r+(g+g m+r f) i \omega-(1+f+m+r) \omega^{2}-i \omega^{3}=0 .
$$

Equating real and imaginary parts of (3.1) gives

$$
2 r(g-f)=\omega^{2}(1+m+r+f), \omega^{2}=(g+g m+r f)
$$

since $\omega \neq 0$. Eliminating $\omega^{2}$ from (3.2) gives $0<m<1, g>f, r>\frac{(1+m)(1+m+f)}{1-m}$, so that

$$
g=g_{0}=\frac{2 r+r(1+m+r+f)}{2 r-(m+1)(1+m+r+f)} f, \quad \omega^{2}=\omega_{0}^{2}=\frac{2 r f(1+m+r)}{2 r-(1+m)(1+m+r+f)}
$$

Eigenvalues of the equation are $\lambda_{1,2}= \pm i \omega_{0}$ and $\lambda_{3}=-(1+f+m+r)$. We therefore have a Hopf bifurcation when $g=g_{0}, 0<m<1, g>f$, and $r>\frac{(m+1)(m+f+1)}{1-m}$.

It is also necessary for us to check the transversality condition for a non-degenerate Hopf bifurcation. Differentiating (2.9) with respect to $g$ gives

$$
\begin{aligned}
\left.\operatorname{Re}\left(\lambda^{\prime}(g)\right)\right|_{g=g_{0}, \lambda=i \omega_{0}} & =-\frac{g_{2}^{2}}{2\left(f^{3}(1+m)-g_{1}^{2}\left(g_{2}+f+f m\right)+f^{2}\left(3(1+m)^{2}+3 m r+r\right)+3 f g_{3}\right)} \\
& \neq 0,
\end{aligned}
$$

where $g_{1}, g_{2}$, and $g_{3}$ can be found in the Appendix. The transversality condition is therefore satisfied. 
Theorem 3.2 Let $0<m<1, r>\frac{(m+1)(m+f+1)}{1-m}$, and $g=g_{0}=\frac{2 r+r(r+f+m+1)}{2 r-(m+1)(r+f+m+1)} f$. If $\left.\operatorname{Re}\left(\lambda^{\prime}(g)\right)\right|_{g=g_{0}, \lambda=i \omega_{0}}>0$ and $\operatorname{Re} C_{1}(0)>0$, then in a sufficiently small left neighborhood of parameter $g_{0}$ bifurcating periodic solutions exist for $\mu_{2}<0$. The periodic solutions of (1.1) at $P^{+}$are non-degenerate, subcritical, and orbitally unstable. The period of the period solution and its characteristic exponent are respectively:

$$
T=\frac{2 \pi}{\omega_{0}}\left(1+\tau_{2} \varepsilon^{2}+o\left(\varepsilon^{4}\right)\right), \quad \beta=\beta_{2} \varepsilon^{2}+o\left(\varepsilon^{4}\right),
$$

where

$$
\begin{aligned}
\omega_{0}= & \frac{\sqrt{2 f r(1+m+r)}}{\sqrt{-1-m^{2}-f(1+m)+r-m(2+r)}}, \\
\tau_{2}= & -\frac{\operatorname{Im} C_{1}(0)+\mu_{2} \omega^{\prime}(0)}{\omega_{0}} \\
= & \frac{1}{64\left(r f g_{1}\right)^{\frac{3}{2}} g_{2}}\left(-A_{5} \sqrt{g_{2}}\left(\sqrt{2} A_{8} r\left(f g_{2}-g_{3}\right)+A_{7} g_{2} \sqrt{g_{1} g_{2} f r}\right)\right. \\
& +A_{6}\left(r A_{7} \sqrt{2 g_{2}}\left(-f g_{2}+g_{3}\right)+A_{8} g_{2}^{2} \sqrt{f r g_{1}}\right) \\
& \left.-16 \sqrt{g_{1}}\left(2 r A_{1} \sqrt{f r}\left(-f g_{2}+g_{3}\right)+g_{2} \sqrt{f r g_{2}}\left(A_{3} \sqrt{g_{2}}-A_{4} \sqrt{g_{2}}+\sqrt{2 r f g_{1}} A_{2}\right)\right)\right), \\
\beta_{2}= & 2 \operatorname{Re} C_{1}(0)=-\frac{\sqrt{2 g_{2}} A_{6} A_{7}+\sqrt{2 g_{2}} A_{5} A_{8}-32 \sqrt{f r g_{1}} A_{1}}{32 \sqrt{f r g_{1}}} \\
\varepsilon^{2}= & \frac{r-r_{0}}{\mu_{2}}+o\left[\left(r-r_{0}\right)^{2}\right], \\
\mu_{2}= & -\frac{\operatorname{Re} C_{1}(0)}{\alpha^{\prime}(0)} \\
= & -\frac{1}{32 \sqrt{f r g_{1}} g_{2}^{2}}\left(\sqrt{2 g_{2}} A_{6} A_{7}+\sqrt{2 g_{2}} A_{5} A_{8}-32 \sqrt{f r g_{1}} A_{1}\right) \\
& \times\left(3 f g_{3}+f^{3}(1+m)-g_{1}^{2}\left(f+g_{2}+f m\right)+f^{2}\left(3+3 m^{2}+r+3 m(2+r)\right)\right), \\
& \\
& \\
&
\end{aligned}
$$

and $A_{1}, A_{2}, A_{3}, A_{4}, A_{5}, A_{6}, A_{7}, A_{8}$ can be found in the Appendix.

Proof When $0<m<1, r>\frac{(m+1)(m+f+1)}{1-m}$, and $g=g_{0}$, equation (2.9) has a negative real root $-(1+f+m+r)$ and a pair of conjugate complex roots, given by Theorem 3.1: $\pm \frac{\sqrt{2 f r(1+m+r)}}{\sqrt{-1-m^{2}-f(1+m)+r-m(2+r)}} i$. Setting $x_{1}=x-\sqrt{1-\frac{f}{g_{0}}}, y_{1}=y-\sqrt{1-\frac{f}{g_{0}}}$, and $z_{1}=z-1$, transforms system (1.1) into

$$
\left\{\begin{array}{l}
\dot{x}_{1}=r\left(y_{1}-x_{1}\right), \\
\dot{y}_{1}=(1+m) x_{1}-(1+m) y_{1}+\sqrt{1-\frac{f}{g_{0}}} z_{1}+x_{1} z_{1}, \\
\dot{z}_{1}=g_{0}(m-1) \sqrt{1-\frac{f}{g_{0}}} x_{1}-g_{0}(m+1) \sqrt{1-\frac{f}{g_{0}}} y_{1}-f z_{1}+g_{0} m x_{1}^{2}-g_{0}(m+1) x_{1} y_{1} .
\end{array}\right.
$$

$E_{0}=(0,0,0)$ is an equilibrium of system (3.3) and the characteristic equation about the $E_{0}=(0,0,0)$ is $(2.9)$ with $g=g_{0}$ :

$$
\lambda^{3}+(1+m+r+f) \lambda^{2}+\left(g_{0}+r f+m g_{0}\right) \lambda+2 g_{0} r-2 r f=0 .
$$

The roots of the above equation are given above in Theorem 3.1. 
The eigenvectors corresponding to $\lambda_{1}, \lambda_{2}, \lambda_{3}$ are $\alpha_{1}, \alpha_{2}, \alpha_{3}$, respectively, where

$$
\alpha_{1,2}=\left(\begin{array}{c}
\frac{g_{2} g_{4} \sqrt{r}}{g_{3}\left(g_{1}+f\right)} \pm i \frac{g_{2} g_{4} \sqrt{g_{1} g_{2}}}{g_{3}\left(g_{1}+f\right) \sqrt{2 f}} \\
-\frac{g_{2} g_{4}(1+m)}{g_{3}\left(g_{1}+f\right) \sqrt{r}} \pm i \frac{g_{4}\left(2 f+g_{2}\right) \sqrt{g_{1} g_{2}}}{g_{3}\left(g_{1}+f\right) \sqrt{2 f}} \\
1
\end{array}\right), \quad \alpha_{3}=\left(\begin{array}{c}
\frac{g_{4} \sqrt{r}}{f\left(g_{1}+f\right)} \\
-\frac{g_{4}(1+f+m)}{f\left(g_{1}+f\right) \sqrt{r}}
\end{array}\right)
$$

and $g_{1}, g_{2}, g_{3}, g_{4}$ are given in the Appendix.

For system (3.3), define

$$
T_{2}=\left(\operatorname{Im} \alpha_{1}, \operatorname{Re} \alpha_{1}, \alpha_{3}\right)=\left(\begin{array}{ccc}
\frac{g_{2} g_{4} \sqrt{g_{1} g_{2}}}{g_{3}\left(g_{1}+f\right) \sqrt{2 f}} & \frac{g_{2} g_{4} \sqrt{r}}{g_{3}\left(g_{1}+f\right)} & \frac{g_{4} \sqrt{r}}{f\left(g_{1}+f\right)} \\
\frac{g_{4}\left(g_{2}+2 f\right) \sqrt{g_{1} g_{2}}}{g_{3}\left(g_{1}+f\right) \sqrt{2 f}} & -\frac{g_{2} g_{4}(1+m)}{g_{3}\left(g_{1}+f\right) \sqrt{r}} & -\frac{g_{4}(1+f+m)}{f\left(g_{1}+f\right) \sqrt{r}} \\
0 & 1 & 1
\end{array}\right)
$$

and $\left(x_{1}, y_{1}, z_{1}\right)^{T}=T_{2}(u, v, w)^{T}$. Then system (3.3) transforms into

$$
\begin{aligned}
& \dot{u}=-\frac{\sqrt{2 f r(1+m+r)}}{\sqrt{-1-m^{2}-f(1+m)+r-m(2+r)}} v+F_{1}(u, v, w), \\
& \dot{v}=\frac{\sqrt{2 f r(1+m+r)}}{\sqrt{-1-m^{2}-f(1+m)+r-m(2+r)}} u+F_{2}(u, v, w), \\
& \dot{w}=-(1+f+m+r) w+F_{3}(u, v, w),
\end{aligned}
$$

where

$$
\begin{aligned}
F_{1}(u, v, w)= & -\frac{f_{2} g_{4} \sqrt{r}\left(f g_{2}-g_{3}\right)(v+w)}{f_{1} g_{3}\left(f+g_{1}\right)}-\frac{f r f_{2} g_{4}^{2}\left(2+f+g_{1}\right)\left(m f_{2}-(1+m) f_{3}\right)}{f_{1} g_{3}\left(f+g_{1}\right)^{2}}, \\
F_{2}(u, v, w)= & \frac{f_{2} g_{2} g_{4} \sqrt{g_{1} g_{2}}(v+w)}{f_{1} g_{3} \sqrt{2 f}\left(f+g_{1}\right)} \\
& +\frac{f_{2} g_{4}^{2} \sqrt{g_{1} r}\left(2+f+g_{1}\right)\left(m f_{2}-(1+m) f_{3}\right)\left(2 f r+g_{2}\left(g_{1}+f\right)\right)}{f_{1} g_{3} \sqrt{2 f g_{2}}\left(f+g_{1}\right)^{2}}, \\
F_{3}(u, v, w)= & -\frac{f_{2} g_{2} g_{4} \sqrt{g_{1} g_{2}}(v+w)}{f_{1} g_{3} \sqrt{2 f}\left(f+g_{1}\right)} \\
& +\frac{f_{2} g_{4}^{2} \sqrt{f r g_{1} g_{2}}\left(2+f+g_{1}\right)\left(f_{3}-m f_{2}+m f_{3}\right)\left(2 f r+g_{1} g_{2}\right)}{\sqrt{2} f_{1} g_{3}^{2}\left(f+g_{1}\right)^{2}},
\end{aligned}
$$

and $f_{1}, f_{2}, f_{3}$ in the Appendix. Furthermore, based on the theory of Hopf bifurcation [9-11], some dominant quantities can be worked out:

$$
\begin{aligned}
& g_{11}=\frac{1}{4}\left[\left(\frac{\partial^{2} F_{1}}{\partial u^{2}}+\frac{\partial^{2} F_{1}}{\partial v^{2}}\right)+i\left(\frac{\partial^{2} F_{2}}{\partial u^{2}}+\frac{\partial^{2} F_{2}}{\partial v^{2}}\right)\right], \\
& g_{02}=\frac{1}{4}\left[\left(\frac{\partial^{2} F_{1}}{\partial u^{2}}-\frac{\partial^{2} F_{1}}{\partial v^{2}}-2 \frac{\partial^{2} F_{2}}{\partial u \partial v}\right)+i\left(\frac{\partial^{2} F_{2}}{\partial u^{2}}-\frac{\partial^{2} F_{2}}{\partial v^{2}}+2 \frac{\partial^{2} F_{1}}{\partial u \partial v}\right)\right], \\
& g_{20}=\frac{1}{4}\left[\left(\frac{\partial^{2} F_{1}}{\partial u^{2}}-\frac{\partial^{2} F_{1}}{\partial v^{2}}+2 \frac{\partial^{2} F_{2}}{\partial u \partial v}\right)+i\left(\frac{\partial^{2} F_{2}}{\partial u^{2}}-\frac{\partial^{2} F_{2}}{\partial v^{2}}-2 \frac{\partial^{2} F_{1}}{\partial u \partial v}\right)\right],
\end{aligned}
$$




$$
\begin{aligned}
G_{21}= & \frac{1}{8}\left(\frac{\partial^{3} F_{1}}{\partial u^{3}}+\frac{\partial^{3} F_{1}}{\partial u \partial v^{2}}+\frac{\partial^{3} F_{2}}{\partial u^{2} \partial v}+\frac{\partial^{3} F_{2}}{\partial v^{3}}\right) \\
& +\frac{1}{8} i\left(\frac{\partial^{3} F_{2}}{\partial u^{3}}+\frac{\partial^{3} F_{2}}{\partial u \partial v^{2}}-\frac{\partial^{3} F_{1}}{\partial u^{2} \partial v}-\frac{\partial^{3} F_{1}}{\partial v^{3}}\right), \\
h_{11}= & \frac{1}{4}\left(\frac{\partial^{2} F_{3}}{\partial u^{2}}+\frac{\partial^{2} F_{3}}{\partial v^{2}}\right), \\
h_{20}= & \frac{1}{4}\left(\frac{\partial^{2} F_{3}}{\partial u^{2}}-\frac{\partial^{2} F_{3}}{\partial v^{2}}-2 i \frac{\partial^{2} F_{3}}{\partial u \partial v}\right),
\end{aligned}
$$

we can obtain the results about $g_{11}, g_{02}, g_{20}, G_{21}$, and $h_{11}, h_{20}$, which can be found in the Appendix.

Solving

$$
\begin{aligned}
& \lambda_{3} \omega_{11}=-h_{11}, \\
& \left(\lambda_{3}-2 i \omega_{0}\right) \omega_{20}=-h_{20},
\end{aligned}
$$

we can get $\omega_{11}$ and $\omega_{20}$ (see the Appendix). Furthermore,

$$
\begin{aligned}
& G_{110}=\frac{1}{2}\left[\left(\frac{\partial^{2} F_{1}}{\partial u \partial w}+\frac{\partial^{2} F_{2}}{\partial v \partial w}\right)+i\left(\frac{\partial^{2} F_{2}}{\partial u \partial w}-\frac{\partial^{2} F_{1}}{\partial v \partial w}\right)\right], \\
& G_{101}=\frac{1}{2}\left[\left(\frac{\partial^{2} F_{1}}{\partial u \partial w}-\frac{\partial^{2} F_{2}}{\partial v \partial w}\right)+i\left(\frac{\partial^{2} F_{2}}{\partial u \partial w}+\frac{\partial^{2} F_{1}}{\partial v \partial w}\right)\right], \\
& g_{21}=G_{21}+\left(2 G_{110} \omega_{11}+G_{101} \omega_{20}\right),
\end{aligned}
$$

which can be found in the Appendix.

Then, some dominant quantities can be worked out:

$$
\begin{aligned}
C_{1}(0)= & \frac{i}{2 \omega_{0}}\left[g_{20} g_{11}-2\left|g_{11}\right|^{2}-\frac{1}{3}\left|g_{02}\right|^{2}\right]+\frac{g_{21}}{2} \\
= & -\frac{\sqrt{2 g_{2}} A_{6} A_{7}+\sqrt{2 g_{2}} A_{5} A_{8}-32 \sqrt{f r g_{1}} A_{1}}{64 \sqrt{f r g_{1}}} \\
& +i \frac{16 \sqrt{2 g_{2}} A_{3}-16 \sqrt{2 g_{2}} A_{4}+\sqrt{2 g_{2}} A_{5} A_{7}-\sqrt{2 g_{2}} A_{6} A_{8}+32 \sqrt{f r g_{1}} A_{2}}{64 \sqrt{f r g_{1}}}, \\
\mu_{2}=- & \frac{\operatorname{Re} C_{1}(0)}{\left.\operatorname{Re}\left(\lambda^{\prime}(g)\right)\right|_{g=g_{0}, \lambda=i \omega_{0}}} \\
=- & \frac{1}{32 \sqrt{f r g_{1}} g_{2}^{2}}\left(\sqrt{2 g_{2}} A_{6} A_{7}+\sqrt{2 g_{2}} A_{5} A_{8}-32 \sqrt{f r g_{1}} A_{1}\right) \\
& \times\left(3 f g_{3}+f^{3}(1+m)-g_{1}^{2}\left(f+g_{2}+f m\right)+f^{2}\left(3+3 m^{2}+r+3 m(2+r)\right)\right), \\
\tau_{2}=- & \frac{\operatorname{Im} C_{1}(0)+\mu_{2} \omega^{\prime}(0)}{\omega_{0}} \\
= & \frac{1}{64\left(r f g_{1}\right)^{\frac{3}{2} g_{2}}}\left(-A_{5} \sqrt{g_{2}}\left(\sqrt{2} A_{8} r\left(f g_{2}-g_{3}\right)+A_{7} g_{2} \sqrt{g_{1} g_{2} f r}\right)\right. \\
+ & A_{6}\left(r A_{7} \sqrt{2 g_{2}}\left(-f g_{2}+g_{3}\right)+A_{8} g_{2}^{2} \sqrt{f r g_{1}}\right)
\end{aligned}
$$




$$
\begin{aligned}
& \left.-16 \sqrt{g_{1}}\left(2 r A_{1} \sqrt{f r}\left(-f g_{2}+g_{3}\right)+g_{2} \sqrt{f r g_{2}}\left(A_{3} \sqrt{g_{2}}-A_{4} \sqrt{g_{2}}+\sqrt{2 r f g_{1}} A_{2}\right)\right)\right), \\
\beta_{2}= & 2 \operatorname{Re} C_{1}(0)=-\frac{\sqrt{2 g_{2}} A_{6} A_{7}+\sqrt{2 g_{2}} A_{5} A_{8}-32 \sqrt{f r g_{1}} A_{1}}{32 \sqrt{f r g_{1}}},
\end{aligned}
$$

where

$$
\begin{aligned}
& \alpha^{\prime}(0)=\operatorname{Re}\left(\lambda^{\prime}\left(g_{0}\right)\right)=-\frac{g_{2}^{2}}{2\left(f^{3}(1+m)-g_{1}^{2}\left(g_{2}+f+f m\right)+f^{2}\left(3(1+m)^{2}+3 m r+r\right)+3 f g_{3}\right)}, \\
& \omega_{0}=\frac{\sqrt{2 f r(1+m+r)}}{\sqrt{-1-m^{2}-f(1+m)+r-m(2+r)}}, \\
& \omega^{\prime}(0)=\frac{r \sqrt{g_{2}}\left(-f g_{2}+g_{3}\right)}{\sqrt{2 r f g_{1}}\left(-g_{1}^{2} g_{2}+3 f g_{3}+f^{3}(1+m)-f g_{1}^{2}(1+m)+f^{2}\left(3+3 m^{2}+r+3 m(2+r)\right)\right)}, \\
& T=\frac{2 \pi}{\omega_{0}}\left(1+\tau_{2} \varepsilon^{2}+o\left(\varepsilon^{4}\right)\right), \\
& \beta=\beta_{2} \varepsilon^{2}+o\left(\varepsilon^{4}\right), \\
& \varepsilon^{2}=\frac{r-r_{0}}{\mu_{2}}+o\left[\left(r-r_{0}\right)^{2}\right] .
\end{aligned}
$$

With the exception of an arbitrary phase angle, the bifurcating periodic solution is

$$
\begin{aligned}
(x, y, z)^{T}= & \left(\sqrt{1-\frac{f}{g_{0}}}, \sqrt{1-\frac{f}{g_{0}}}, 1\right)^{T}+\left(\begin{array}{ccc}
\frac{g_{2} g_{4} \sqrt{g_{1} g_{2}}}{g_{3}\left(g_{1}+f\right) \sqrt{2 f}} & \frac{g_{2} g_{4} \sqrt{r}}{g_{3}\left(g_{1}+f\right)} & \frac{g_{4} \sqrt{r}}{f\left(g_{1}+f\right)} \\
\frac{g_{4}\left(g_{2}+2 f\right) \sqrt{g_{1} g_{2}}}{g_{3}\left(g_{1}+f\right) \sqrt{2 f}} & -\frac{g_{2} g_{4}(1+m)}{g_{3}\left(g_{1}+f\right) \sqrt{r}} & -\frac{g_{4}(1+f+m)}{f\left(g_{1}+f\right) \sqrt{r}} \\
0 & 1 & 1
\end{array}\right) \\
& \times(u, v, w)^{T}
\end{aligned}
$$

where

$$
\begin{aligned}
& u=\operatorname{Re} \theta, \quad v=\operatorname{Im} \theta, \quad w=\omega_{11}|\theta|^{2}+\operatorname{Re}\left(\omega_{20} \theta^{2}\right)+O\left(|\theta|^{3}\right), \\
& \theta=\varepsilon e^{2 i t \pi / T}+\frac{i \varepsilon^{2}}{6 \omega_{0}}\left[g_{02} e^{-4 i t \pi / T}-3 g_{02} e^{4 i t \pi / T}+6 g_{11}\right]+O\left(\varepsilon^{3}\right) .
\end{aligned}
$$

Because the system is symmetry about the $\mathrm{z}$ axis, Theorems 3.1 and 3.2 are also true for $P^{-}\left(-\sqrt{1-\frac{f}{g_{0}}},-\sqrt{1-\frac{f}{g_{0}}}, 1\right)$.

\section{Darboux integrability of system (1.1)}

Let $R[x, y, z]$ be the ring of the real polynomials in the variables $x, y$, and $z$. We say that $h(x, y, z) \in R(x, y, z)$ is a Darboux polynomial of system (1.1) if it satisfies

$$
\frac{\partial h}{\partial x} P+\frac{\partial h}{\partial y} Q+\frac{\partial h}{\partial z} R=h L_{h}
$$

for some polynomial $L_{h}$, which is called the cofactor of $h(x, y, z)$ [17-28]. If $h(x, y, z)$ is a Darboux polynomial, then the surface $h(x, y, z)=0$ is an invariant algebraic surface [18-21, $25,28]$, which means that if an orbit of system (1.1) has a point on the surface $h(x, y, z)=0$, 
then the whole orbit is contained in it. We assert categorically that the order $k$ of $L_{h}$ is at most to 1 . This is because

$$
\begin{aligned}
& \operatorname{deg}\left(L_{h}\right)+\operatorname{deg}(h) \\
& \quad=\max \{\operatorname{deg}(h)-1+\operatorname{deg}(P), \operatorname{deg}(h)-1+\operatorname{deg}(Q), \operatorname{deg}(h)-1+\operatorname{deg}(R)\} \\
& \quad \leq \operatorname{deg}(h)+1 .
\end{aligned}
$$

Assume that the cofactor is

$$
L_{h}=b_{0}+b_{1} x+b_{2} y+b_{3} z
$$

then (4.1) becomes

$$
\frac{\partial h}{\partial x} P+\frac{\partial h}{\partial y} Q+\frac{\partial h}{\partial z} R=h\left(b_{0}+b_{1} x+b_{2} y+b_{3} z\right)
$$

where $b_{0}, b_{1}, b_{2}, b_{3} \in R$.

Supposing that $E=e^{\frac{g}{h}}$ is an exponential factor of (1.1). Thus, $h, g$ both are Darboux polynomials of equations (1.1) and $h, g$ are coprime [22-28]. If real-valued polynomial $L_{e}$ is the cofactor of $E$, then

$$
\frac{\partial E}{\partial x} P+\frac{\partial E}{\partial y} Q+\frac{\partial E}{\partial z} R=E L_{e} .
$$

If $H(x, y, z)$ is a first integral [26, 27], then

$$
\frac{\partial H}{\partial x} P+\frac{\partial H}{\partial y} Q+\frac{\partial H}{\partial z} R=0
$$

Theorem 4.1 When $r \neq 0$, the following four conclusions are suitable for equations (1.1).

(1) There are no Darboux polynomials with cofactors not equal to zero.

(2) Their polynomial first integrals do not exist.

(3) Their exponential factors cannot be found.

(4) Their first integrals of Darboux type also do not exist.

Proof Since there are four parts to the proof of Theorem 4.1, we consider two cases of $p=0$ and $p \neq 0$ for each of these four parts in turn.

Part (1). Let

$$
h(x, y, z)=\sum_{i=0}^{n} h_{i}
$$

where $h_{i}=h_{i}(x, y, z)$ is a polynomial and its each item is of degree $i$. Assume $h_{n} \neq 0, n \neq 0$.

Collecting the terms of degree $n+1$ in (4.3) yields

$$
x z \frac{\partial h_{n}}{\partial y}+g\left(m x^{2}-(1+m) x y\right) \frac{\partial h_{n}}{\partial z}=h_{n}\left(b_{1} x+b_{2} y+b_{3} z\right) .
$$


Solving this equation for $h_{n}$, we can get

$$
\begin{aligned}
h_{n}(x, y, z)= & F\left(x,-2 g m x y+g(m+1) y^{2}+z^{2}\right) \\
& \times \exp \left(b_{3} \frac{y}{x}-\frac{b_{1}}{\sqrt{g(1+m)}} k(x, y, z)-b_{2} \frac{z \sqrt{g(1+m)}+x g m k(x, y, z)}{\sqrt{g(1+m)} g(1+m) x}\right),
\end{aligned}
$$

where $F$ is a smooth function and

$$
k(x, y, z)=\arctan \left(\frac{\sqrt{g(1+m)}(m x-(1+m) y)}{(1+m) z}\right) .
$$

For the assumption of $h_{n}(x, y, z)$, we have $b_{1}=b_{2}=b_{3}=0$.

Let

$$
h_{n}(x, y, z)=x^{n-2 p}\left(g(1+m) y^{2}-2 g m x y+z^{2}\right)^{p} \text {, }
$$

where the (nonnegative) integer $p$ satisfies $p \leq \frac{n}{2}$.

Calculating the terms of order $n$ in (4.3), one can obtain

$$
\begin{aligned}
& x z \frac{\partial h_{n-1}}{\partial y}+g x(m(x-y)-y) \frac{\partial h_{n-1}}{\partial z}+r(y-x) \frac{\partial h_{n}}{\partial x}+(m(x-y)-y) \frac{\partial h_{n}}{\partial y}-\frac{\partial h_{n}}{\partial z} f z \\
& \quad=b_{0} h_{n} .
\end{aligned}
$$

Case 1. $p=0$. Solving equation (4.10), one can obtain

$$
\begin{aligned}
h_{n-1}(x, y, z)= & F\left(x,-2 g m x y+g(m+1) y^{2}+z^{2}\right)-\frac{x^{n-1} k(x, y, z)}{\sqrt{g(1+m)}} b_{0} \\
& -\frac{n r x^{n-2}(g x k(x, y, z)-\sqrt{g(1+m)} z)}{\sqrt{g(1+m)} g(1+m)},
\end{aligned}
$$

where $F$ is a smooth function. Considering the definition of polynomial, we must have $n=0, b_{0}=0$, which is impossible.

Case 2. $p>0$. Solving equation (4.10), one can obtain

$$
\begin{aligned}
h_{n-1}(x, y, z)= & F\left(x,-2 g m x y+g(m+1) y^{2}+z^{2}\right)-b_{0} \frac{k(x, y, z)}{x \sqrt{g(1+m)}} h_{n} \\
& -\frac{z y}{(1+m)^{2}} k_{1} k_{2}-k \frac{(1+m) r n+p k_{3} h_{n}+k_{1} k_{4} m^{2} x^{3} g}{x \sqrt{g m+m}(1+m)^{2}} \\
& +\frac{z}{g(1+m)^{2} x^{2}}\left((m+1)(n r-2 p r) h_{n}+k_{1} k_{5} g m x^{3}\right),
\end{aligned}
$$

where $F$ is a smooth function and

$$
\begin{aligned}
& k_{1}(x, y, z)=p x^{n-2 p-1}\left(-2 g m x y+(g m+g) y^{2}+z^{2}\right)^{p-1}, \\
& k_{2}(x, y, z)=m^{3}+(-f+r+3) m^{2}+(-2 f+r+3) m-f+1, \\
& k_{3}(x, y, z)=m^{3}+(f+r+3) m^{2}+(2 f-r+3) m+f-2 r+1,
\end{aligned}
$$




$$
\begin{aligned}
& k_{4}(x, y, x)=m^{2}+(f+r+2) m+(f-2 r+1), \\
& k_{5}(x, y, z)=m^{2}+(-f-r+2) m+(-f+2 r+1) .
\end{aligned}
$$

Since $h_{n-1}(x, y, z)$ is a polynomial, we obtain $p=0, n=0, b_{0}=0$. And the case is again not possible.

Part (2). Let $b_{0}=b_{1}=b_{2}=b_{3}=0$ in (4.3). Then $h$ satisfies

$$
\frac{\partial h}{\partial x} P+\frac{\partial h}{\partial y} Q+\frac{\partial h}{\partial z} R=0
$$

We still assume

$$
h=\sum_{i=0}^{n} h_{i}(x, y, z)
$$

where $h_{i}=h_{i}(x, y, z)$ is a polynomial and its each item is of degree $i$. Assume that $h_{n} \neq 0$ and $n \neq 0$.

Collecting terms of degree $n+1$ in (4.11) yields

$$
x z \frac{\partial h_{n}}{\partial y}+g\left(m x^{2}-(1+m) x y\right) \frac{\partial h_{n}}{\partial z}=0 .
$$

Solving this equation for $h_{n}$ gives

$$
h_{n}(x, y, z)=F\left(x,-2 g m x y+g(m+1) y^{2}+z^{2}\right) \text {, }
$$

where $F$ is a smooth function.

Assume

$$
h_{n}(x, y, z)=x^{n-2 p}\left(g(1+m) y^{2}-2 g m x y+z^{2}\right)^{p},
$$

where $p$ is a nonnegative integer and $p \leq \frac{n}{2}$.

Terms of degree $n$ in (4.11) are

$$
x z \frac{\partial h_{n-1}}{\partial y}+g x(m(x-y)-y) \frac{\partial h_{n-1}}{\partial z}+r(y-x) \frac{\partial h_{n}}{\partial x}+(m(x-y)-y) \frac{\partial h_{n}}{\partial y}-\frac{\partial h_{n}}{\partial z} f z=0
$$

Case 1. Solving equation (4.14) for $p=0$, we can give

$$
\begin{aligned}
h_{n-1}(x, y, z)= & F\left(x,-2 g m x y+g(m+1) y^{2}+z^{2}\right) \\
& -\frac{n r x^{n-2}(g x k(x, y, z)-\sqrt{g(1+m)} z)}{\sqrt{g(1+m)} g(1+m)},
\end{aligned}
$$

where $F$ is a smooth function. Since the expression of $h_{n-1}(x, y, z)$, it forces $n=0$, which is illogicality, because one has made the assumption that $n \neq 0$. This hypothesis therefore does not hold. 
Case 2. Solving equation (4.14) for $p>0$, we can give

$$
\begin{aligned}
h_{n-1}(x, y, z)= & F\left(x,-2 g m x y+g(m+1) y^{2}+z^{2}\right)-\frac{k_{7}(x, y, z) y z}{(1+m)^{2}} \\
& +\frac{(m+1)(n-2 p) r z}{g x^{2}(1+m)^{2}} h_{n}+\frac{m x z g_{5} k_{5}(x, y, z)}{(1+m)^{2}} \\
& -\frac{\left.k(x, y, z)\left((m+1) r n+k_{6}(x, y, z) p\right) h_{n}+g m^{2} x^{3} k_{4}(x, y, z) g_{5}\right)}{x \sqrt{g m+g}(1+m)^{2}}
\end{aligned}
$$

for a smooth function $F$. Also

$$
\begin{aligned}
& k_{6}(x, y, z)=m^{3}+1+m^{2} r-m r-2 r+(1+m)^{2} f+3 m(m+1), \\
& k_{7}(x, y, z)=m^{3}+(-f+r+3) m^{2}+(-2 f+r+3) m-f+1 .
\end{aligned}
$$

Thus, $p=0, n=0$, there is no possibility, because in the above, one has assumed that $n \neq 0$. So this hypothesis again does not hold.

Part (3). By Theorem 4.1(1), one can make an assumption that equations (1.1) have an exponential factor $E=x^{u}$ with $u \in R[x, y, z]$. Suppose that the cofactor $L=b_{0}+b_{1} x+b_{2} y+$ $b_{3} z$ with $b_{i} \in R$ for $i=0,1,2,3$.

Obviously, by (4.4), $u$ is the solution of equation (4.15), that is to say,

$$
\frac{\partial u}{\partial x} P+\frac{\partial u}{\partial y} Q+\frac{\partial u}{\partial z} R=b_{0}+b_{1} x+b_{2} y+b_{3} z
$$

We can write $u$ as

$$
u=\sum_{i=0}^{n} u_{i}(x, y, z)
$$

where each $u_{i}=u_{i}(x, y, z)$ is homogeneous of degree $i$. Supposing that $u_{n} \neq 0$ and $n \neq 0$ again, calculating terms of order $n+1$ in (4.15), we can have

$$
x z \frac{\partial u_{n}}{\partial y}+g\left(m x^{2}-(1+m) x y\right) \frac{\partial u_{n}}{\partial z}=0
$$

Solving this equation for $u_{n}$ gives

$$
u_{n}(x, y, z)=F\left(x,-2 g m x y+g(m+1) y^{2}+z^{2}\right)
$$

for a smooth function $F$.

Assume

$$
u_{n}(x, y, z)=x^{n-2 p}\left(g(1+m) y^{2}-2 g m x y+z^{2}\right)^{p},
$$

where $p$ is a nonnegative integer and $p \leq \frac{n}{2}$.

Calculating terms of order $n$ in (4.15) gets

$$
x z \frac{\partial u_{n-1}}{\partial y}+g x(m(x-y)-y) \frac{\partial u_{n-1}}{\partial z}+r(y-x) \frac{\partial u_{n}}{\partial x}+(m(x-y)-y) \frac{\partial u_{n}}{\partial y}-\frac{\partial u_{n}}{\partial z} f z=0
$$


Case 1. When $p=0$, equation (4.18) yields

$$
\begin{aligned}
u_{n-1}(x, y, z)= & F\left(x,-2 g m x y+g(m+1) y^{2}+z^{2}\right) \\
& -\frac{n r x^{n-2}(g x k(x, y, z)-\sqrt{g(1+m)} z)}{\sqrt{g(1+m)} g(1+m)}
\end{aligned}
$$

for a smooth function $F$. Thus, one can obtain that $n=0$, which is also impossible for $n \neq 0$ in the assumption. This hypothesis cannot hold.

Case 2. Solving equation (4.18) for $p>0$ yields

$$
\begin{aligned}
u_{n-1}(x, y, z)= & F\left(x,-2 g m x y+g(m+1) y^{2}+z^{2}\right)-\frac{k_{7}(x, y, z) y z}{(1+m)^{2}} \\
& +\frac{(m+1)(n-2 p) r z}{g x^{2}(1+m)^{2}} u_{n}+\frac{m x z g_{5} k_{5}(x, y, z)}{(1+m)^{2}} \\
& -\frac{k(x, y, z)\left(\left((m+1) r n+k_{6}(x, y, z) p\right) u_{n}+g m^{2} x^{3} k_{4}(x, y, z) g_{5}\right)}{x \sqrt{g m+g}(1+m)^{2}},
\end{aligned}
$$

where $F$ is a smooth function. Since $u_{n-1}(x, y, z)$ is a polynomial, we get $p=0, n=0$, contradicting the assumption that $n \neq 0$. This hypothesis again does not hold.

Part (4). By the arguments of Part (3) above, together with the definition of the exponential factor, Part (4) follows.

\section{Conclusions}

The dynamic properties of equations (1.1), which describe the segmented disc dynamo with mechanical friction, are investigated extensively. We determined the equilibria and their linear stabilities. We analyzed the direction of Hopf bifurcations and determined the stability of the bifurcating limit cycles by using the theory of normal form in detail. Some numerical simulations are presented to illustrate the theoretical analysis. In addition, the Darboux integrability of the system is studied. It is expected that more detailed theory analysis and simulation investigations will be provided in a forthcoming study.

\section{Appendix}

$$
\begin{aligned}
g_{4}= & \sqrt{\frac{(1+m+r)(1+f+m+r)}{3+f+m+r}}, \\
f_{1}= & -\frac{\sqrt{g_{1} g_{2}} g_{2}^{2} g_{4}^{2}(1+m)}{\sqrt{2 f r}\left(f+g_{1}\right)^{2} g_{3}^{2}}+\frac{\sqrt{g_{1} g_{2}} g_{2} g_{4}^{2}(1+f+m)}{f g_{3} \sqrt{2 f r}\left(f+g_{1}\right)^{2}} \\
& -\frac{\sqrt{g_{1} g_{2} r} g_{2} g_{4}^{2}\left(2 f+g_{2}\right)}{\sqrt{2 f} g_{3}^{2}\left(f+g_{1}\right)^{2}}+\frac{\sqrt{g_{1} g_{2} r}\left(2 f+g_{2}\right) g_{4}^{2}}{f g_{3} \sqrt{2 f}\left(f+g_{1}\right)^{2}}, \\
f_{2}= & \frac{\sqrt{g_{1} g_{2}} g_{2} g_{4} u}{\sqrt{2 f}\left(f+g_{1}\right) g_{3}}+\frac{g_{2} g_{4} \sqrt{r} v}{g_{3}\left(f+g_{1}\right)}+\frac{g_{4} \sqrt{r} w}{f\left(f+g_{1}\right)}, \\
f_{3}= & \frac{\sqrt{g_{1} g_{2}} g_{4}\left(2 f+g_{2}\right) u}{\sqrt{2 f} g_{3}\left(f+g_{1}\right)}-\frac{g_{2} g_{4}(1+m) v}{g_{3}\left(f+g_{1}\right) \sqrt{r}}-\frac{g_{4}(1+f+m) w}{f\left(f+g_{1}\right) \sqrt{r}}, \\
f_{4}= & -\frac{\sqrt{r g_{1} g_{2}} g_{1} g_{2}\left(2 f+g_{2}\right)}{\sqrt{2 f}\left(f+g_{1}\right)\left(2+f+g_{1}\right) g_{3}^{2}}+\frac{\sqrt{g_{1} g_{2}} g_{4}^{2}\left(g_{1} g_{2} g_{3}+f\left(g_{2} g_{3}-g_{2}^{2}(1+m)+2 r g_{3}\right)\right)}{\sqrt{2 f r} f\left(f+g_{1}\right)^{2} g_{3}^{2}},
\end{aligned}
$$




$$
\begin{aligned}
& f_{5}=-\frac{g_{2} g_{4}^{2}(1+f+m)}{f\left(f+g_{1}\right)^{2} g_{3}}+\frac{g_{1} g_{2}(1+m)}{f\left(f+g_{1}\right)\left(2+f+g_{1}\right) g_{3}}, \\
& g_{11}=\frac{1}{4}\left(-\frac{2 r g_{2} g_{4}^{2}\left(f g_{2}-g_{3}\right)}{f_{4}\left(f+g_{1}\right)^{2} g_{3}^{2}}-\frac{r g_{2} f_{5} g_{1}^{2}\left(g_{2}+2 f(1+m)\right)}{f_{4}\left(f+g_{1}\right) g_{3}^{2}}\right. \\
& +\frac{2 r f g_{2} f_{5}\left(\left(2+f+g_{1}\right)(1+m)^{2} g_{4}^{2}+m r g_{1}\left(f+g_{1}\right)\right)}{f_{4}\left(f+g_{1}\right)^{2} g_{3}^{2}} \\
& +i\left(\frac{\sqrt{2 r g_{1} g_{2}} g_{1} g_{2}^{2}}{\sqrt{f} f_{4}\left(f+g_{1}\right)\left(2+f+g_{1}\right) g_{3}^{2}}-\frac{\sqrt{r g_{1} g_{2}} g_{1}^{2} g_{2} g_{4}^{2}\left(g_{2}+2 f(1+m)\right)\left(2 f r+g_{2}\left(g_{1}+f\right)\right)}{\sqrt{2 f f f_{4} g_{3}^{3}\left(f+g_{1}\right)^{3}}}\right. \\
& \left.\left.+\frac{\sqrt{2 r g_{1} g_{2}} g_{2} g_{4}^{2}\left(\left(2+f+g_{1}\right)(1+m)^{2} g_{4}^{2}+g_{1} m r\left(f+g_{1}\right)\right)\left(2 f r+g_{2}\left(g_{1}+f\right)\right)}{\sqrt{r} f_{4}\left(f+g_{1}\right)^{4} g_{3}^{3}}\right)\right), \\
& g_{02}=\frac{1}{4}\left(\frac{2 g_{2}\left(f g_{2}-g_{3}\right) g_{4}^{2} r}{f_{4}\left(f+g_{1}\right)^{2} g_{3}^{2}}-\frac{2 r f f_{5} g_{2}\left(\left(2+f+g_{1}\right)(1+m)^{2} g_{4}^{2}+g_{1}\left(f+g_{1}\right) m r\right)}{f_{4}\left(f+g_{1}\right)^{2} g_{3}^{2}}\right. \\
& -\frac{f_{5} g_{1}^{2} g_{2}\left(g_{2}+2 f(1+m)\right) r}{f_{4}\left(f+g_{1}\right) g_{3}^{2}}-2\left(\frac{g_{1}^{2} g_{2}^{3}}{2 f f_{4} g_{3}^{2}\left(f+g_{1}\right)\left(2+f+g_{1}\right)}\right. \\
& +\frac{\sqrt{g_{1} g_{2}} g_{4}^{2}\left(2 f r+g_{2}\left(g_{1}+f\right)\right)}{\sqrt{2 r f f} f_{4} g_{3}\left(f+g_{1}\right)^{2}}
\end{aligned}
$$

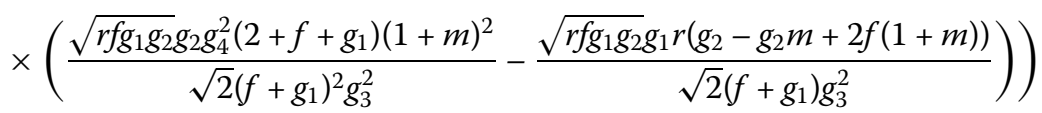

$$
\begin{aligned}
& +i\left(-\frac{\sqrt{2 r g_{1} g_{2}} g_{1} g_{2}^{2}}{\sqrt{f} f_{4}\left(f+g_{1}\right)\left(2+f+g_{1}\right) g_{3}^{2}}-\frac{\sqrt{r g_{1} g_{2}} g_{1}^{2} g_{2} g_{4}^{2}\left(g_{2}+2 f(1+m)\right)\left(2 f r+g_{2}\left(g_{1}+f\right)\right)}{\sqrt{2 f f f_{4}}\left(f+g_{1}\right)^{3} g_{3}^{3}}\right. \\
& -\frac{\sqrt{2 r g_{1} g_{2}} g_{2} g_{4}^{2}\left(\left(2+f+g_{1}\right)(1+m)^{2} g_{4}^{2}+m r g_{1}\left(f+g_{1}\right)\right)\left(2 f r+g_{2}\left(g_{1}+f\right)\right)}{\sqrt{f} f_{4}\left(f+g_{1}\right)^{4} g_{3}^{3}} \\
& +2\left(-\frac{\sqrt{r g_{1} g_{2}} g_{2} g_{4}^{2}\left(f g_{2}-g_{3}\right)}{\sqrt{2 f} f f_{4}\left(f+g_{1}\right)^{2} g_{3}^{2}}-f_{5}\left(-\frac{\sqrt{r f g_{1} g_{2}} g_{2} g_{4}^{2}\left(2+f+g_{1}\right)(1+m)^{2}}{\sqrt{2} f_{4}\left(f+g_{1}\right)^{2} g_{3}^{2}}\right.\right. \\
& \left.\left.\left.\left.+\frac{\sqrt{r f g_{1} g_{2}} g_{1} r\left(g_{2}-g_{2} m+2 f(1+m)\right)}{\sqrt{2} f_{4}\left(f+g_{1}\right) g_{3}^{2}}\right)\right)\right)\right) \text {, } \\
& g_{20}=\frac{1}{4}\left(\frac{2 g_{2}\left(f g_{2}-g_{3}\right) g_{4}^{2} r}{f_{4}\left(f+g_{1}\right)^{2} g_{3}^{2}}-\frac{2 r f f_{5} g_{2}\left(\left(2+f+g_{1}\right)(1+m)^{2} g_{4}^{2}+g_{1}\left(f+g_{1}\right) m r\right)}{f_{4}\left(f+g_{1}\right)^{2} g_{3}^{2}}\right. \\
& -\frac{f_{5} g_{1}^{2} g_{2}\left(g_{2}+2 f(1+m)\right) r}{f_{4}\left(f+g_{1}\right) g_{3}^{2}}+2\left(\frac{g_{1}^{2} g_{2}^{3}}{2 f_{4} g_{3}^{2}\left(f+g_{1}\right)\left(2+f+g_{1}\right)}\right. \\
& +\frac{\sqrt{g_{1} g_{2}} g_{4}^{2}\left(2 f r+g_{2}\left(g_{1}+f\right)\right)}{\sqrt{2 r f} f f_{4} g_{3}\left(f+g_{1}\right)^{2}}
\end{aligned}
$$

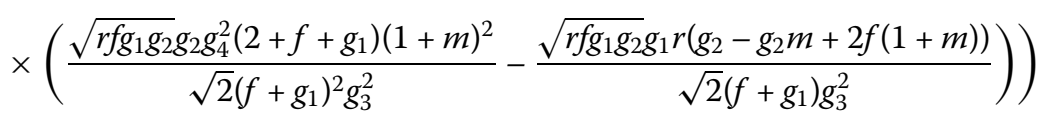

$$
\begin{aligned}
& +i\left(-\frac{\sqrt{2 r g_{1} g_{2}} g_{1} g_{2}^{2}}{\sqrt{f} f_{4}\left(f+g_{1}\right)\left(2+f+g_{1}\right) g_{3}^{2}}-\frac{\sqrt{r g_{1} g_{2}} g_{1}^{2} g_{2} g_{4}^{2}\left(g_{2}+2 f(1+m)\right)\left(2 f r+g_{2}\left(g_{1}+f\right)\right)}{\sqrt{2 f f f_{4}}\left(f+g_{1}\right)^{3} g_{3}^{3}}\right. \\
& -\frac{\sqrt{2 r g_{1} g_{2}} g_{2} g_{4}^{2}\left(\left(2+f+g_{1}\right)(1+m)^{2} g_{4}^{2}+m r g_{1}\left(f+g_{1}\right)\right)\left(2 f r+g_{2}\left(g_{1}+f\right)\right)}{\sqrt{f} f_{4}\left(f+g_{1}\right)^{4} g_{3}^{3}} \\
& +2\left(\frac{\sqrt{r g_{1} g_{2}} g_{2} g_{4}^{2}\left(f g_{2}-g_{3}\right)}{\sqrt{2 f} f_{4}\left(f+g_{1}\right)^{2} g_{3}^{2}}+f_{5}\left(-\frac{\sqrt{r f g_{1} g_{2}} g_{2} g_{4}^{2}\left(2+f+g_{1}\right)(1+m)^{2}}{\sqrt{2} f_{4}\left(f+g_{1}\right)^{2} g_{3}^{2}}\right.\right.
\end{aligned}
$$




$$
\left.\left.\left.+\frac{\sqrt{r f g_{1} g_{2}} g_{1} r\left(g_{2}-g_{2} m+2 f(1+m)\right)}{\sqrt{2} f_{4}\left(f+g_{1}\right) g_{3}^{2}}\right)\right)\right),
$$

$G_{21}=0$

$$
\begin{aligned}
& h_{11}=\frac{1}{4}\left(-\frac{\sqrt{2 r g_{1} g_{2}} g_{1} g_{2}^{2}}{\sqrt{f} f_{4} g_{3}^{2}\left(f+g_{1}\right)\left(2+f+g_{1}\right)}\right. \\
& +\left(\frac{\sqrt{g_{1} g_{2}} g_{2}^{2} g_{4}^{2}(1+m)}{\sqrt{2 f r} g_{3}^{2}\left(f+g_{1}\right)^{2}}+\frac{\sqrt{r g_{1} g_{2}} g_{1} g_{2}\left(2 f+g_{2}\right)}{\sqrt{2 f} g_{3}^{2}\left(f+g_{1}\right)\left(2+f+g_{1}\right)}\right) \\
& \left.\times\left(\frac{r g_{1} g_{2}\left(g_{1} g_{2}+2 f\left(g_{1}+m g_{1}-m r\right)\right)}{f_{4} g_{3}^{2}\left(f+g_{1}\right)}-\frac{2 r f g_{2} g_{4}^{2}\left(2+f+g_{1}\right)(1+m)^{2}}{f_{4} g_{3}^{2}\left(f+g_{1}\right)^{2}}\right)\right), \\
& h_{20}=\frac{1}{4}\left(\frac{\sqrt{2 r g_{1} g_{2}} g_{1} g_{2}^{2}}{\sqrt{f} f_{4} g_{3}^{2}\left(f+g_{1}\right)\left(2+f+g_{1}\right)}\right. \\
& +\left(\frac{\sqrt{g_{1} g_{2}} g_{2}^{2} g_{4}^{2}(1+m)}{\sqrt{2 f r} g_{3}^{2}\left(f+g_{1}\right)^{2}}+\frac{\sqrt{r g_{1} g_{2}} g_{1} g_{2}\left(2 f+g_{2}\right)}{\sqrt{2 f} g_{3}^{2}\left(f+g_{1}\right)\left(2+f+g_{1}\right)}\right) \\
& \times\left(\frac{r g_{1} g_{2}\left(g_{1} g_{2}+2 f\left(g_{1}+m g_{1}+m r\right)\right)}{f_{4} g_{3}^{2}\left(f+g_{1}\right)}+\frac{2 r f g_{2} g_{4}^{2}\left(2+f+g_{1}\right)(1+m)^{2}}{f_{4} g_{3}^{2}\left(f+g_{1}\right)^{2}}\right) \\
& +2 i\left(\frac{g_{1}^{2} g_{2}^{3}}{2 f f_{4} g_{3}^{2}\left(f+g_{1}\right)\left(2+f+g_{1}\right)}\right. \\
& +\left(\frac{\sqrt{g_{1} g_{2}} g_{2}^{2} g_{4}^{2}(1+m)}{\sqrt{2 f r} g_{3}^{2}\left(f+g_{1}\right)^{2}}+\frac{\sqrt{r g_{1} g_{2}} g_{1} g_{2}\left(2 f+g_{2}\right)}{\sqrt{2 f} g_{3}^{2}\left(f+g_{1}\right)\left(2+f+g_{1}\right)}\right) \\
& \left.\times\left(\frac{\sqrt{r f g_{1} g_{2}} g_{2} g_{4}^{2}\left(2+f+g_{1}\right)(1+m)^{2}}{\sqrt{2} f_{4} g_{3}^{2}\left(f+g_{1}\right)^{2}}-\frac{\sqrt{r f g_{1} g_{2}} r g_{1}\left(g_{2}-g_{2} m+2 f(1+m)\right)}{\sqrt{2} f_{4} g_{3}^{2}\left(f+g_{1}\right)}\right)\right), \\
& \omega_{11}=\frac{1}{4\left(f+g_{1}\right)}\left(-\frac{\sqrt{2 r g_{1} g_{2}} g_{1} g_{2}^{2}}{\sqrt{f} f_{4} g_{3}^{2}\left(f+g_{1}\right)\left(2+f+g_{1}\right)}\right. \\
& +\left(\frac{\sqrt{g_{1} g_{2}} g_{2}^{2} g_{4}^{2}(1+m)}{\sqrt{2 f r}\left(f+g_{1}\right)^{2} g_{3}^{2}}+\frac{\sqrt{r g_{1} g_{2}} g_{1} g_{2}\left(2 f+g_{2}\right)}{\sqrt{2 f}\left(f+g_{1}\right)\left(2+f+g_{1}\right) g_{3}^{2}}\right) \\
& \left.\times\left(\frac{g_{1} g_{2} r\left(g_{1} g_{2}+2 f\left(g_{1}+g_{1} m-m r\right)\right)}{f_{4}\left(f+g_{1}\right) g_{3}^{2}}-\frac{2 f r g_{2} g_{4}^{2}\left(2+f+g_{1}\right)(1+m)^{2}}{f_{4} g_{3}^{2}\left(f+g_{1}\right)^{2}}\right)\right), \\
& \omega_{20}=\frac{f g_{2}+g_{1} g_{2}}{4\left(f^{2} g_{2}+g_{1}^{2} g_{2}+2 f g_{1}\left(g_{2}+4 r\right)\right)}\left(\frac{\sqrt{2 r g_{1} g_{2}} g_{1} g_{2}^{2}}{\sqrt{f} f_{4} g_{3}^{2}\left(f+g_{1}\right)\left(2+f+g_{1}\right)}\right. \\
& +\left(\frac{\sqrt{g_{1} g_{2}} g_{2}^{2} g_{4}^{2}(1+m)}{\sqrt{2 f r}\left(f+g_{1}\right)^{2} g_{3}^{2}}+\frac{\sqrt{r g_{1} g_{2}} g_{1} g_{2}\left(2 f+g_{2}\right)}{\sqrt{2 f}\left(f+g_{1}\right)\left(2+f+g_{1}\right) g_{3}^{2}}\right) \\
& \left.\times\left(\frac{g_{1} g_{2} r\left(g_{1} g_{2}+2 f\left(g_{1}+g_{1} m+m r\right)\right)}{f_{4}\left(f+g_{1}\right) g_{3}^{2}}+\frac{2 f r g_{2} g_{4}^{2}\left(2+f+g_{1}\right)(1+m)^{2}}{f_{4} g_{3}^{2}\left(f+g_{1}\right)^{2}}\right)\right) \\
& +\frac{\sqrt{2 f r g_{1} g_{2}}}{\left(f^{2} g_{2}+g_{1}^{2} g_{2}+2 f g_{1}\left(g_{2}+4 r\right)\right)}\left(\frac{g_{1}^{2} g_{2}^{3}}{2 f f_{4} g_{3}^{2}\left(f+g_{1}\right)\left(2+f+g_{1}\right)}\right. \\
& +\left(\frac{\sqrt{g_{1} g_{2}} g_{2}^{2} g_{4}^{2}(1+m)}{\sqrt{2 f r}\left(f+g_{1}\right)^{2} g_{3}^{2}}+\frac{\sqrt{r g_{1} g_{2}} g_{1} g_{2}\left(2 f+g_{2}\right)}{\sqrt{2 f}\left(f+g_{1}\right)\left(2+f+g_{1}\right) g_{3}^{2}}\right) \\
& \left.\times\left(\frac{\sqrt{f r g_{1} g_{2}} g_{2} g_{4}^{2}\left(2+f+g_{1}\right)(1+m)^{2}}{\sqrt{2} f_{4}\left(f+g_{1}\right)^{2} g_{3}^{2}}-\frac{\sqrt{f r g_{1} g_{2}} g_{1} r\left(g_{2}-g_{2} m+2 f(1+m)\right)}{\sqrt{2} f_{4} g_{3}^{2}\left(f+g_{1}\right)}\right)\right)
\end{aligned}
$$




$$
\begin{aligned}
& +i\left(\frac { f g _ { 2 } + g _ { 1 } g _ { 2 } } { 2 ( f ^ { 2 } g _ { 2 } + g _ { 1 } ^ { 2 } g _ { 2 } + 2 f g _ { 1 } ( g _ { 2 } + 4 r ) ) } \left(\frac{g_{1}^{2} g_{2}^{3}}{2 f f_{4} g_{3}^{2}\left(f+g_{1}\right)\left(2+f+g_{1}\right)}\right.\right. \\
& +\left(\frac{\sqrt{g_{1} g_{2}} g_{2}^{2} g_{4}^{2}(1+m)}{\sqrt{2 f r}\left(f+g_{1}\right)^{2} g_{3}^{2}}+\frac{\sqrt{r g_{1} g_{2}} g_{1} g_{2}\left(2 f+g_{2}\right)}{\sqrt{2 f}\left(f+g_{1}\right)\left(2+f+g_{1}\right) g_{3}^{2}}\right) \\
& \left.\times\left(\frac{\sqrt{f r g_{1} g_{2}} g_{2} g_{4}^{2}\left(2+f+g_{1}\right)(1+m)^{2}}{\sqrt{2} f_{4}\left(f+g_{1}\right)^{2} g_{3}^{2}}-\frac{\sqrt{f r g_{1} g_{2}} g_{1} r\left(g_{2}-g_{2} m+2 f(1+m)\right)}{\sqrt{2} f_{4} g_{3}^{2}\left(f+g_{1}\right)}\right)\right) \\
& -\frac{\sqrt{2 f r g_{1} g_{2}}}{2\left(f^{2} g_{2}+g_{1}^{2} g_{2}+2 f g_{1}\left(g_{2}+4 r\right)\right)}\left(\frac{\sqrt{2 r g_{1} g_{2}} g_{1} g_{2}^{2}}{\sqrt{f} f_{4} g_{3}^{2}\left(f+g_{1}\right)\left(2+f+g_{1}\right)}\right. \\
& +\left(\frac{\sqrt{g_{1} g_{2}} g_{2}^{2} g_{4}^{2}(1+m)}{\sqrt{2 f r}\left(f+g_{1}\right)^{2} g_{3}^{2}}+\frac{\sqrt{r g_{1} g_{2}} g_{1} g_{2}\left(2 f+g_{2}\right)}{\sqrt{2 f}\left(f+g_{1}\right)\left(2+f+g_{1}\right) g_{3}^{2}}\right) \\
& \left.\left.\times\left(\frac{g_{1} g_{2} r\left(g_{1} g_{2}+2 f\left(g_{1}+g_{1} m+m r\right)\right)}{f_{4}\left(f+g_{1}\right) g_{3}^{2}}+\frac{2 f r g_{2} g_{4}^{2}\left(2+f+g_{1}\right)(1+m)^{2}}{f_{4} g_{3}^{2}\left(f+g_{1}\right)^{2}}\right)\right)\right), \\
& G_{110}=\frac{1}{2}\left(\frac{\sqrt{r g_{1} g_{2}} g_{1} g_{2}^{2}}{\sqrt{2 f} f_{4}\left(f+g_{1}\right)\left(2+f+g_{1}\right) g_{3}^{2}}-\frac{\sqrt{r g_{1} g_{2}} g_{2} g_{4}^{2}\left(f g_{2}-2 g_{3}\right)}{\sqrt{2 f f f} f_{4}\left(f+g_{1}\right)^{2} g_{3}^{2}}\right. \\
& +\left(\frac{g_{1} g_{2}(1+m)}{f_{4}\left(f+g_{1}\right)\left(2+f+g_{1}\right) g_{3}}-\frac{g_{2} g_{4}^{2}(1+f+m)}{f_{4}\left(f+g_{1}\right)^{2} g_{3}^{2}}\right) \\
& \times\left(\frac{\sqrt{r g_{1}} g_{4}^{2}\left(2+f+g_{1}\right)\left(-2 f(1+m) r+g_{2}\left(1+f+2 m+f m+m^{2}-r+m r\right)\right)}{\sqrt{2 f g_{2} g_{3}}\left(f+g_{1}\right)^{2}}\right) \\
& +\frac{\sqrt{g_{1} g_{2}} g_{4}^{2}\left(2 f r+g_{2}\left(g_{1}+f\right)\right.}{\sqrt{2 r f} f f_{4}\left(f+g_{1}\right)^{2} g_{3}} \\
& \left.\times\left(\frac{r g_{1}(1+m)^{2}}{\left(f+g_{1}\right) g_{3}}+\frac{\left.r g_{4}^{2}\left(2+f+g_{1}\right)\right)\left(1+m^{2}+f(1+m)+2 m(1+r)\right)}{\left(f+g_{1}\right)^{2} g_{3}}\right)\right) \\
& +\frac{i}{2}\left(\frac{g_{1}^{2} g_{2}^{3}}{2 f f_{4}\left(f+g_{1}\right)\left(2+f+g_{1}\right) g_{3}^{2}}+\frac{\left(f^{2} g_{2}^{2}-g_{3}^{2}\right) g_{4}^{2} r}{f^{2} f_{4}\left(f+g_{1}\right)^{2} g_{3}^{2}}+\frac{\sqrt{g_{1} g_{2}} g_{4}^{2}\left(2 f r+g_{2}\left(g_{1}+f\right)\right)}{\not f_{4} g_{3} \sqrt{2 r f}\left(f+g_{1}\right)^{2}}\right. \\
& \times\left(\frac{\sqrt{r g_{1}} g_{4}^{2}\left(2+f+g_{1}\right)\left(-2 r f(1+m)-g_{2}^{2}\right)}{\sqrt{2 f g_{2}}\left(f+g_{1}\right)^{2} g_{3}}\right) \\
& -\left(\frac{g_{1} g_{2}(1+m)}{f g_{3}\left(f+g_{1}\right)\left(2+f+g_{1}\right)}-\frac{g_{2} g_{4}^{2}(1+f+m)}{f\left(f+g_{1}\right)^{2} g_{3}}\right) \\
& \left.\times\left(\frac{r g_{1}(1+m)^{2}}{f_{4}\left(f+g_{1}\right) g_{3}}+\frac{r g_{4}^{2}\left(2+f+g_{1}\right)\left(1+m^{2}+f(1+m)+2 m(1+r)\right)}{g_{3} f_{4}\left(f+g_{1}\right)^{2}}\right)\right), \\
& G_{101}=\frac{1}{2}\left(-\frac{\sqrt{r g_{1} g_{2}} g_{1} g_{2}^{2}}{\sqrt{2 f} f_{4} g_{3}^{2}\left(f+g_{1}\right)\left(2+f+g_{1}\right)}-\frac{\sqrt{r g_{1} g_{2}} g_{2}^{2} g_{4}^{2}}{\sqrt{2 f} f_{4} g_{3}^{2}\left(f+g_{1}\right)^{2}}\right. \\
& +\left(\frac{g_{1} g_{2}(1+m)}{f_{4} g_{3}\left(f+g_{1}\right)\left(2+f+g_{1}\right)}-\frac{g_{2} g_{4}^{2}(1+f+m)}{f_{4} g_{3}\left(f+g_{1}\right)^{2}}\right) \\
& \times \frac{\sqrt{r g_{1}} g_{4}^{2}\left(2+f+g_{1}\right)\left(-2 r f(1+m)-g_{1} g_{2}\right)}{\sqrt{2 f g_{2}} g_{3}\left(f+g_{1}\right)^{2}}-\frac{\sqrt{g_{1} g_{2}} g_{4}^{2}\left(2 r f+g_{2}\left(g_{1}+f\right)\right)}{\sqrt{2 r f} f g_{3} f_{4}\left(f+g_{1}\right)^{2}} \\
& \left.\times\left(\frac{r g_{1}(1+m)^{2}}{g_{3}\left(f+g_{1}\right)}+\frac{r g_{4}^{2}\left(2+f+g_{1}\right)\left(1+m^{2}+f(1+m)+2 m(1+r)\right)}{\left(f+g_{1}\right)^{2} g_{3}}\right)\right) \\
& +\frac{i}{2}\left(\frac{g_{1}^{2} g_{2}^{3}}{2 f_{4}\left(f+g_{1}\right)\left(2+f+g_{1}\right) g_{3}^{2}}-\frac{r g_{4}^{2}\left(f^{2} g_{2}^{2}-g_{3}^{2}\right)}{f^{2} f_{4}\left(f+g_{1}\right)^{2} g_{3}^{2}}+\frac{\sqrt{g_{1} g_{2}} g_{4}^{2}\left(2 f r+g_{2}\left(g_{1}+f\right)\right)}{\sqrt{2 r f} f_{4} g_{3}\left(f+g_{1}\right)^{2}}\right.
\end{aligned}
$$




$$
\begin{aligned}
& \times \frac{\sqrt{r g_{1}} g_{4}^{2}\left(2+f+g_{1}\right)\left(-2 f r(1+m)-g_{1} g_{2}\right)}{\sqrt{2 f g_{2}} g_{3}\left(f+g_{1}\right)^{2}} \\
& +\left(\frac{g_{1} g_{2}(1+m)}{f g_{3}\left(f+g_{1}\right)\left(2+f+g_{1}\right)}-\frac{g_{2} g_{4}^{2}(1+f+m)}{f\left(f+g_{1}\right)^{2} g_{3}}\right) \\
& \left.\times\left(\frac{r g_{1}(1+m)^{2}}{g_{3} f_{4}\left(f+g_{1}\right)}+\frac{r g_{4}^{2}\left(2+f+g_{1}\right)\left(1+m^{2}+f(1+m)+2 m(1+r)\right)}{f_{4} g_{3}\left(f+g_{1}\right)^{2}}\right)\right), \\
& g_{211}=-\frac{1}{4\left(f+g_{1}\right)}\left(\frac{\sqrt{2 r g_{1} g_{2}} g_{1} g_{2}^{2}}{\sqrt{f} f_{4} g_{3}^{2}\left(f+g_{1}\right)\left(2+f+g_{1}\right)}\right. \\
& -\frac{r g_{1}^{2} g_{2}\left(g_{2}+2 f(1+m)\right)}{g_{3}^{2}\left(f+g_{1}\right)}\left(\frac{\sqrt{g_{1} g_{2}} g_{2}^{2} g_{4}^{2}(1+m)}{\sqrt{2 r f f} f_{4} g_{3}^{2}\left(f+g_{1}\right)^{2}}\right. \\
& \left.+\frac{\sqrt{r g_{1} g_{2}} g_{1} g_{2}\left(2 f+g_{2}\right)}{\sqrt{2 f} f_{4} g_{3}^{2}\left(f+g_{1}\right)\left(2+f+g_{1}\right)}\right)+\left(\frac{\sqrt{g_{1} g_{2}} g_{2}^{2} g_{4}^{2}(1+m)}{\sqrt{2 r f}\left(f+g_{1}\right)^{2} g_{3}^{2}}\right. \\
& \left.\left.+\frac{\sqrt{r g_{1} g_{2}} g_{1} g_{2}\left(2 f+g_{2}\right)}{\sqrt{2 f} g_{3}^{2}\left(f+g_{1}\right)\left(2+f+g_{1}\right)}\right)\left(\frac{2 r g_{2} g_{4}^{2}\left(2+f+g_{1}\right)(1+m)^{2}}{f_{4} g_{3}^{2}\left(f+g_{1}\right)^{2}}+\frac{2 m r^{2} f g_{1} g_{2}}{f_{4}\left(f+g_{1}\right) g_{3}^{2}}\right)\right), \\
& g_{212}=\frac{\sqrt{r g_{1} g_{2}} g_{1} g_{2}^{2}}{\sqrt{2 f} f_{4} g_{3}^{2}\left(f+g_{1}\right)\left(2+f+g_{1}\right)}-\frac{\sqrt{r g_{1} g_{2}} g_{2} g_{4}^{2}\left(f g_{2}-2 g_{3}\right)}{\sqrt{2 f f f_{4}}\left(f+g_{1}\right)^{2} g_{3}^{2}} \\
& +\left(\frac{g_{1} g_{2}(1+m)}{f_{4} g_{3}\left(f+g_{1}\right)\left(2+f+g_{1}\right)}-\frac{g_{2} g_{4}^{2}(1+f+m)}{f_{4} g_{3}\left(f+g_{1}\right)^{2}}\right) \\
& \times\left(-\frac{\sqrt{r g_{1}} r g_{4}^{2}\left(2+f+g_{1}\right)\left(2 f+g_{2}\right)(1+m)}{\sqrt{2 f g_{2}} g_{3}\left(f+g_{1}\right)^{2}}\right. \\
& \left.+\frac{\sqrt{r g_{1} g_{2}} g_{4}^{2}\left(2+f+g_{1}\right)\left(1+m^{2}+f(1+m)+2 m(1+r)\right)}{\sqrt{2 f} g_{3}^{2}\left(f+g_{1}\right)^{2}}\right) \\
& \times \frac{\sqrt{g_{1} g_{2}} g_{4}^{2}\left(2 f r+g_{2}\left(g_{1}+f\right)\right)}{\sqrt{2 r f f f f_{4} g_{3}\left(f+g_{1}\right)^{2}}}\left(\frac{r g_{1}(1+m)^{2}}{g_{3}\left(f+g_{1}\right)}\right. \\
& \left.+\frac{r g_{4}^{2}\left(2+f+g_{1}\right)\left(1+m^{2}+f(1+m)+2 m(1+r)\right)}{g_{3}\left(f+g_{1}\right)^{2}}\right), \\
& g_{213}=\frac{g_{1}^{2} g_{2}^{3}}{2 f_{4} g_{3}^{2}\left(f+g_{1}\right)\left(2+f+g_{1}\right)}+\frac{r g_{2} g_{4}^{2}\left(f g_{2}-g_{3}\right)}{f f g_{3}^{2}\left(f+g_{1}\right)^{2}} \\
& +\frac{r g_{4}^{2}\left(f g_{2}-g_{3}\right)}{f^{2} f_{4} g_{3}\left(f+g_{1}\right)^{2}}+\frac{\sqrt{g_{1} g_{2}} g_{4}^{2}\left(2 r f+g_{2}\left(g_{1}+f\right)\right)}{\sqrt{2 r f f f} f_{4} g_{3}\left(f+g_{1}\right)^{2}} \\
& \times\left(-\frac{r g_{4}^{2} \sqrt{r g_{1}}\left(2+f+g_{1}\right)\left(2 f+g_{2}\right)(1+m)}{\sqrt{2 f g_{2}} g_{3}\left(f+g_{1}\right)^{2}}\right. \\
& \left.+\frac{\sqrt{r g_{1} g_{2}} g_{4}^{2}\left(2+f+g_{1}\right)\left(1+m^{2}+f(1+m)+2 m(1+r)\right)}{\sqrt{2 f} g_{3}\left(f+g_{1}\right)^{2}}\right) \\
& \times\left(\frac{g_{1} g_{2}(1+m)}{f g_{3}\left(f+g_{1}\right)\left(2+f+g_{1}\right)}-\frac{g_{2} g_{4}^{2}(1+f+m)}{f g_{3}\left(f+g_{1}\right)^{2}}\right)\left(\frac{r g_{1}(1+m)^{2}}{g_{3} f_{4}\left(f+g_{1}\right)}\right. \\
& \left.+\frac{r g_{4}^{2}\left(2+f+g_{1}\right)\left(1+m^{2}+f(1+m)+2 m(1+r)\right)}{f_{4} g_{3}\left(f+g_{1}\right)^{2}}\right), \\
& g_{214}=-\frac{\sqrt{2 r g_{1} g_{2}} g_{1} g_{2}^{2}}{\sqrt{f} f_{4} g_{3}^{2}\left(f+g_{1}\right)\left(2+f+g_{1}\right)}-\frac{r g_{1}^{2} g_{2}\left(g_{2}+2 f(1+m)\right)}{g_{3}^{2}\left(f+g_{1}\right)}\left(\frac{\sqrt{g_{1} g_{2}} g_{2}^{2} g_{4}^{2}(1+m)}{\sqrt{2 r f} f_{4} g_{3}^{2}\left(f+g_{1}\right)^{2}}\right.
\end{aligned}
$$




$$
\begin{aligned}
& \left.+\frac{\sqrt{r g_{1} g_{2}} g_{1} g_{2}\left(2 f+g_{2}\right)}{\sqrt{2 f} f_{4} g_{3}^{2}\left(f+g_{1}\right)\left(2+f+g_{1}\right)}\right) \\
& -\left(\frac{\sqrt{g_{1} g_{2}} g_{2}^{2} g_{4}^{2}(1+m)}{\sqrt{2 r f} g_{3}^{2}\left(f+g_{1}\right)^{2}}+\frac{\sqrt{r g_{1} g_{2}} g_{1} g_{2}\left(2 f+g_{2}\right)}{\sqrt{2 f} g_{3}^{2}\left(f+g_{1}\right)\left(2+f+g_{1}\right)}\right) \\
& \times\left(\frac{2 r f g_{2} g_{4}^{2}\left(2+f+g_{1}\right)(1+m)^{2}}{f_{4} g_{3}^{2}\left(f+g_{1}\right)^{2}}+\frac{2 f m r^{2} g_{1} g_{2}}{f_{4}\left(f+g_{1}\right) g_{3}^{2}}\right), \\
& g_{215}=\frac{g_{1}^{2} g_{2}^{3}}{2 f_{4} g_{3}^{2}\left(f+g_{1}\right)\left(2+f+g_{1}\right)}+\left(\frac{\sqrt{g_{1} g_{2}} g_{2}^{2} g_{4}^{2}(1+m)}{\sqrt{2 r f}\left(f+g_{1}\right)^{2} g_{3}^{2}}+\frac{\sqrt{r g_{1} g_{2}} g_{1} g_{2}\left(2 f+g_{2}\right)}{\sqrt{2 f} g_{3}^{2}\left(f+g_{1}\right)\left(2+f+g_{1}\right)}\right) \\
& \times\left(\frac{\sqrt{r f g_{1} g_{2}} g_{2} g_{4}^{2}\left(2+f+g_{1}\right)(1+m)^{2}}{\sqrt{2} f_{4} g_{3}^{2}\left(f+g_{1}\right)^{2}}-\frac{\sqrt{r f g_{1} g_{2}} r g_{1}\left(g_{2}-g_{2} m+2 f(1+m)\right)}{\sqrt{2} f_{4} g_{3}^{2}\left(f+g_{1}\right)}\right), \\
& g_{216}=-\frac{\sqrt{r g_{1} g_{2}} g_{1} g_{2}^{2}}{\sqrt{2 f} f_{4} g_{3}^{2}\left(f+g_{1}\right)\left(2+f+g_{1}\right)}-\frac{\sqrt{r g_{1} g_{2}} g_{2} g_{4}^{2}\left(f g_{2}-g_{3}\right)}{\sqrt{2 f f f} f_{4} g_{3}^{2}\left(f+g_{1}\right)^{2}}-\frac{\sqrt{r g_{1} g_{2}} g_{2} g_{4}^{2}}{\sqrt{2 f f f f_{4} g_{3}\left(f+g_{1}\right)^{2}}} \\
& +\left(\frac{g_{1} g_{2}(1+m)}{f_{4} g_{3}\left(f+g_{1}\right)\left(2+f+g_{1}\right)}-\frac{g_{2} g_{4}^{2}(1+f+m)}{f f_{4} g_{3}\left(f+g_{1}\right)^{2}}\right) \\
& \times\left(-\frac{r g_{4}^{2} \sqrt{r g_{1}}\left(2+f+g_{1}\right)\left(2 f+g_{2}\right)(1+m)}{\sqrt{2 f g_{2}} g_{3}\left(f+g_{1}\right)^{2}}\right. \\
& \left.+\frac{\sqrt{r g_{1} g_{2}} g_{4}^{2}\left(2+f+g_{1}\right)\left(1+m^{2}+f(1+m)+2 m(1+r)\right)}{\sqrt{2 f} g_{3}\left(f+g_{1}\right)^{2}}\right) \\
& -\frac{\sqrt{g_{1} g_{2}} g_{4}^{2}\left(2 r f+g_{2}\left(g_{1}+f\right)\right)}{\sqrt{2 r f} f_{4} g_{3}\left(f+g_{1}\right)^{2}} \\
& +\left(\frac{r g_{1}(1+m)^{2}}{g_{3}\left(f+g_{1}\right)}+\frac{r g_{4}^{2}\left(2+f+g_{1}\right)\left(1+m^{2}+f(1+m)+2 m(1+r)\right)}{g_{3}\left(f+g_{1}\right)^{2}}\right), \\
& g_{217}=\frac{g_{1}^{2} g_{2}^{3}}{2 f f_{4} g_{3}^{2}\left(f+g_{1}\right)\left(2+f+g_{1}\right)}-\frac{r g_{2} g_{4}^{2}\left(f g_{2}-g_{3}\right)}{f_{4} g_{3}^{2}\left(f+g_{1}\right)^{2}}-\frac{r g_{4}^{2}\left(f g_{2}-g_{3}\right)}{f^{2} f_{4} g_{3}\left(f+g_{1}\right)^{2}} \\
& +\frac{\sqrt{g_{1} g_{2}} g_{4}^{2}\left(2 r f+g_{2}\left(g_{1}+f\right)\right)}{\sqrt{2 r f} f f_{4} g_{3}\left(f+g_{1}\right)^{2}}\left(-\frac{\sqrt{r g_{1}} r g_{4}^{2}\left(2+f+g_{1}\right)\left(2 f+g_{2}\right)(1+m)}{\sqrt{2 f g_{2}} g_{3}\left(f+g_{1}\right)^{2}}\right. \\
& \left.+\frac{\sqrt{r g_{1} g_{2}} g_{4}^{2}\left(2+f+g_{1}\right)\left(1+m^{2}+f(1+m)+2 m(1+r)\right)}{\sqrt{2 f} g_{3}\left(f+g_{1}\right)^{2}}\right) \\
& +\left(\frac{g_{1} g_{2}(1+m)}{f g_{3}\left(f+g_{1}\right)\left(2+f+g_{1}\right)}-\frac{g_{2} g_{4}^{2}(1+f+m)}{f g_{3}\left(f+g_{1}\right)^{2}}\right) \\
& \times\left(\frac{r g_{1}(1+m)^{2}}{g_{3} f_{4}\left(f+g_{1}\right)}+\frac{r g_{4}^{2}\left(2+f+g_{1}\right)\left(1+m^{2}+f(1+m)+2 m(1+r)\right)}{f_{4} g_{3}\left(f+g_{1}\right)^{2}}\right) . \\
& g_{21}=g_{211} g_{212}-\frac{\left(2+g_{1}\right)\left(g_{214} g_{216}+2 g_{215} g_{217}\right) g_{2}+4 \sqrt{r g_{1} g_{2}}\left(2 g_{215} g_{216}-g_{214} g_{217}\right)}{8\left(\left(2+g_{1}\right)^{2} g_{2}+16 r g_{1}\right)} \\
& +i\left(g_{211} g_{213}+\frac{\left(2+g_{1}\right)\left(2 g_{215} g_{216}-g_{214} g_{217}\right) g_{2}+4 \sqrt{r g_{1} g_{2}}\left(g_{214} g_{216}+2 g_{215} g_{217}\right)}{8\left(\left(2+g_{1}\right)^{2} g_{2}+16 r g_{1}\right)}\right), \\
& A_{1}=g_{211} g_{212}-\frac{g_{2}\left(2+g_{1}\right)\left(g_{214} g_{216}+2 g_{215} g_{217}\right)+4 \sqrt{g_{1} g_{2} r}\left(2 g_{215} g_{216}-g_{214} g_{217}\right)}{8\left(\left(2+g_{1}\right)^{2} g_{2}+16 r g_{1}\right)} \text {, } \\
& A_{2}=g_{211} g_{213}+\frac{g_{2}\left(2+g_{1}\right)\left(2 g_{215} g_{216}-g_{214} g_{217}\right)+4 \sqrt{g_{1} g_{2} r}\left(g_{214} g_{216}+2 g_{215} g_{217}\right)}{8\left(\left(2+g_{1}\right)^{2} g_{2}+16 r g_{1}\right)},
\end{aligned}
$$




$$
\begin{aligned}
& A_{3}=-\frac{1}{16 r f^{3} g_{3}^{6} f_{4}^{2}\left(f+g_{1}\right)^{8}\left(2+f+g_{1}\right)^{2}}\left(2 r f g _ { 3 } ^ { 2 } ( f + g _ { 1 } ) ^ { 4 } ( 2 + f + g _ { 1 } ) ^ { 2 } \left(-2 r g_{2} g_{4}^{2}\left(f g_{2}-g_{3}\right)\right.\right. \\
& -r f f_{5} g_{1}^{2} g_{2}\left(f+g_{1}\right)\left(g_{2}+2 f(1+m)\right) \\
& \left.+2 r f^{2} f_{5} g_{2}\left(g_{4}^{2}\left(2+f+g_{1}\right)(1+m)^{2}+m r g_{1}\left(f+g_{1}\right)\right)\right)^{2}+g_{1} g_{2}\left(2 r f g_{1} g_{2}^{2} g_{3}\left(f+g_{1}\right)^{3}\right. \\
& -r g_{1}^{2} g_{2} g_{4}^{2}\left(f+g_{1}\right)\left(2+f+g_{1}\right)\left(g_{2}+2 f(1+m)\right)\left(2 f r+g_{2}\left(g_{1}+f\right)\right) \\
& \left.\left.+2 r f g_{2} g_{4}^{2}\left(2+f+g_{1}\right)\left(g_{4}^{2}\left(2+f+g_{1}\right)(1+m)^{2}+m r g_{1}\left(f+g_{1}\right)\right)\left(2 f r+g_{2}\left(g_{1}+f\right)\right)\right)^{2}\right), \\
& A_{4}=\frac{1}{96 r f^{3} f_{4}^{2} g_{3}^{6}\left(f+g_{1}\right)^{8}\left(2+f+g_{1}\right)^{2}}\left(2 f r \left(g_{1}^{2} g_{2}^{3} g_{3}\left(f+g_{1}\right)^{3}\right.\right. \\
& -2 r g_{2} g_{3} g_{4}^{2}\left(f+g_{1}\right)^{2}\left(2+f+g_{1}\right)\left(f g_{2}-g_{3}\right) \\
& +r f f_{5} g_{1}^{2} g_{2} g_{3}\left(f+g_{1}\right)^{3}\left(2+f+g_{1}\right)\left(g_{2}+2 f(1+m)\right)+2 r f^{2} f_{5} g_{2} g_{3}\left(f+g_{1}\right)^{2}\left(2+f+g_{1}\right) \\
& \times\left(g_{4}^{2}\left(2+f+g_{1}\right)(1+m)^{2}+\operatorname{mrg}_{1}\left(f+g_{1}\right)\right) \\
& +g_{1} g_{2} g_{4}^{2}\left(2+f+g_{1}\right)\left(g_{2} g_{4}^{2}\left(2+f+g_{1}\right)(1+m)^{2}\right. \\
& \left.\left.-r g_{1}\left(f+g_{1}\right)\left(g_{2}-g_{2} m+2 f(1+m)\right)\right)\left(2 r f+g_{2}\left(g_{1}+f\right)\right)\right)^{2}+g_{1} g_{2}\left(2 r f g_{1} g_{2}^{2} g_{3}\left(f+g_{1}\right)^{3}\right. \\
& +r g_{1}^{2} g_{2} g_{4}^{2}\left(f+g_{1}\right)\left(2+f+g_{1}\right)\left(g_{2}+2 f(1+m)\right)\left(2 r f+g_{2}\left(g_{1}+f\right)\right) \\
& +2 r f g_{2} g_{4}^{2}\left(2+f+g_{1}\right)\left(g_{4}^{2}\left(2+f+g_{1}\right)(1+m)^{2}+m r g_{1}\left(f+g_{1}\right)\right)\left(2 f r+g_{2}\left(g_{1}+f\right)\right) \\
& +2 r g_{3}\left(f+g_{1}\right)^{2}\left(2+f+g_{1}\right)\left(g_{2} g_{4}^{2}\left(f g_{2}-g_{3}\right)-f^{2} f_{5}\left(g_{2} g_{4}^{2}\left(2+f+g_{1}\right)(1+m)^{2}\right.\right. \\
& \left.\left.\left.\left.-g_{1}\left(f+g_{1}\right)\left(g_{2}-g_{2} m+2 f(1+m)\right) r\right)\right)\right)^{2}\right) \text {, } \\
& A_{5}=-\frac{2 r g_{2} g_{4}^{2}\left(f g_{2}-g_{3}\right)}{f f_{4} g_{3}^{2}\left(f+g_{1}\right)^{2}}+\frac{2 r f f_{5} g_{2}\left(g_{4}^{2}\left(2+f+g_{1}\right)(1+m)^{2}+m r g_{1}\left(f+g_{1}\right)\right)}{f_{4} g_{3}^{2}\left(f+g_{1}\right)^{2}} \\
& -\frac{r f_{5} g_{1}^{2} g_{2}\left(g_{2}+2 f(1+m)\right)}{f_{4} g_{3}^{2}\left(f+g_{1}\right)} \\
& A_{6}=\frac{\sqrt{2 r g_{1} g_{2}} g_{1} g_{2}^{2}}{\sqrt{f} f_{4} g_{3}^{2}\left(f+g_{1}\right)\left(2+f+g_{1}\right)}-\frac{\sqrt{r g_{1} g_{2}} g_{1}^{2} g_{2} g_{4}^{2}\left(g_{2}+2 f(1+m)\right)\left(2 f r+g_{2}\left(g_{1}+f\right)\right)}{\sqrt{2 f} f f_{4} g_{3}^{3}\left(f+g_{1}\right)^{3}} \\
& +\frac{\sqrt{2 r g_{1} g_{2}} g_{2} g_{4}^{2}\left(g_{4}^{2}\left(2+f+g_{1}\right)(1+m)^{2}+m r g_{1}\left(f+g_{1}\right)\right)\left(2 f r+g_{2}\left(g_{1}+f\right)\right)}{\sqrt{f} f_{4} g_{3}^{3}\left(f+g_{1}\right)^{4}}, \\
& A_{7}=\frac{2 r g_{2} g_{4}^{2}\left(f g_{2}-g_{3}\right)}{f_{4} g_{3}^{2}\left(f+g_{1}\right)^{2}}-\frac{r f_{5} g_{1}^{2} g_{2}\left(g_{2}+2 f(1+m)\right)}{f_{4}\left(f+g_{1}\right) g_{3}^{2}} \\
& -\frac{2 r f f_{5} g_{2}\left(g_{4}^{2}\left(2+f+g_{1}\right)(1+m)^{2}+m r g_{1}\left(f+g_{1}\right)\right)}{f_{4} g_{3}^{2}\left(f+g_{1}\right)^{2}} \\
& +2\left(\frac{g_{1}^{2} g_{2}^{3}}{2 f f_{4} g_{3}^{2}\left(f+g_{1}\right)\left(2+f+g_{1}\right)}+\frac{\sqrt{g_{1} g_{2}} g_{4}^{2}\left(2 f r+g_{2}\left(g_{1}+f\right)\right)}{\sqrt{2 f r} f f_{4} g_{3}\left(f+g_{1}\right)^{2}}\right. \\
& \left.\times\left(\frac{\sqrt{f r g_{1} g_{2}} g_{2} g_{4}^{2}\left(2+f+g_{1}\right)(1+m)^{2}}{\sqrt{2} g_{3}^{2}\left(f+g_{1}\right)^{2}}-\frac{\sqrt{f r g_{1} g_{2}} r g_{1}\left(g_{2}-g_{2} m+2 f(1+m)\right)}{\sqrt{2} g_{3}^{2}\left(f+g_{1}\right)}\right)\right), \\
& A_{8}=-\frac{\sqrt{2 r g_{1} g_{2}} g_{1} g_{2}^{2}}{\sqrt{f} f_{4} g_{3}^{2}\left(f+g_{1}\right)\left(2+f+g_{1}\right)}-\frac{\sqrt{r g_{1} g_{2}} g_{1}^{2} g_{2} g_{4}^{2}\left(g_{2}+2 f(1+m)\right)\left(2 r f+g_{2}\left(g_{1}+f\right)\right)}{\sqrt{2 f f f_{4} g_{3}^{3}\left(f+g_{1}\right)^{3}}} \\
& \times \frac{\sqrt{2 r g_{1} g_{2}} g_{2} g_{4}^{2}\left(g_{4}^{2}\left(2+f+g_{1}\right)(1+m)^{2}+m r g_{1}\left(f+g_{1}\right)\right)\left(2 f r+g_{2}\left(g_{1}+f\right)\right)}{\sqrt{f} f_{4} g_{3}^{3}\left(f+g_{1}\right)^{4}}
\end{aligned}
$$




$$
\begin{aligned}
& \left.+2\left(\frac{\sqrt{r g_{1} g_{2}} g_{2} g_{4}^{2}\left(f g_{2}-g_{3}\right)}{\sqrt{2 f f f_{4} g_{3}^{2}\left(f+g_{1}\right)^{2}}+f_{5}\left(-\frac{\sqrt{r f g_{1} g_{2}} g_{2} g_{4}^{2}\left(2+f+g_{1}\right)(1+m)^{2}}{\sqrt{2} f_{4} g_{3}^{2}\left(f+g_{1}\right)^{2}}\right.}+\frac{\sqrt{r f g_{1} g_{2}} r g_{1}\left(g_{2}-g_{2} m+2 f(1+m)\right)}{\sqrt{2} f_{4} g_{3}^{2}\left(f+g_{1}\right)}\right)\right) .
\end{aligned}
$$

\section{Acknowledgements}

The authors would like to express many thanks to Professor Jianghong Bao for her corrections and comments on this manuscript.

\section{Funding}

Zhouchao Wei is supported by the Natural Science Foundation of China (No. 11772306 and No. 11401543), the Open Foundation for Guangxi Colleges and Universities Key Lab of Complex System Optimization and Big Data Processing (No. 2016CSOBDP0202), Scientific Research Program of Hubei Provincial Department of Education (No. B2017599), the Fundamental Research Funds for the Central Universities and China University of Geosciences (Wuhan) (No. CUGL150419).

\section{Availability of data and materials}

Not applicable.

\section{Competing interests}

The authors declare that they have no competing interests.

\section{Authors' contributions}

The authors have made the same contribution. All authors read and approved the final manuscript.

\section{Author details}

'School of Mathematics and Physics, China University of Geosciences, Wuhan, P.R. China. ${ }^{2}$ Guangxi Colleges and Universities Key Laboratory of Complex System Optimization and Big Data Processing, Yulin Normal University, Yulin, P.R. China. ${ }^{3}$ Mathematical Institute, University of Oxford, Oxford, United Kingdom.

\section{Publisher's Note}

Springer Nature remains neutral with regard to jurisdictional claims in published maps and institutional affiliations.

Received: 14 April 2018 Accepted: 28 May 2018 Published online: 11 July 2018

\section{References}

1. Dudkowski, D., Jafari, S., Kapitaniak, T., Kuznetsov, N.V., Leonov, G.A., Prasad, A.: Hidden attractors in dynamical systems. Phys. Rep. 637, 1-50 (2016)

2. Bullard, E.C.: The stability of a homopolar dynamo. Proc. Camb. Philos. Soc. 51, 744-760 (1955)

3. Hide, R:: How to locate the electrically-conducting fluid core a planet from external magnetic observations. Nature 271,640-641 (1978)

4. Knobloch, E.: Chaos in the segmented disc dynamo. Phys. Lett. A 82, 439-440 (1981)

5. Moffatt, H.K.: A self-consistent treatment of simple dynamo systems. Geophys. Astrophys. Fluid Dyn. 14, 147-166 (1979)

6. Moffatt, H.K.: Magnetic Field Generation in Electrically Conducting Fluids. Cambridge University Press, Cambridge (1978)

7. Plunian, F., Marty, P.H., Alemany, A.: Chaotic behaviour of the Rikitake dynamo with symmetric mechanical friction and azimuthal currents. Proc. R. Soc. Lond. A 454, 1835-1842 (1998)

8. Wei, Z.C., Moroz, I., Sprott, J.C., Akgul, A., Zhang, W.: Hidden hyperchaos and electronic circuit application in a 5D self-exciting homopolar disc dynamo. Chaos 27, 033101 (2017)

9. Li, C.B., Sprott, J.C.: Multistability in the Lorenz system: a broken butterfly. Int. J. Bifurc. Chaos 24(10), 1450131 (2014)

10. Li, C.B., Sprott, J.C., Xing, H.Y.: Crisis in amplitude control hides in multistability. Int. J. Bifurc. Chaos 26(14), 1650233 (2016)

11. Wei, Z.C., Moroz, I., Sprott, J.C., Wang, Z., Zhang, W.: Detecting hidden chaotic regions and complex dynamics in the self-exciting homopolar disc dynamo. Int. J. Bifurc. Chaos 27(2), 1730008 (2017)

12. Plunian, F., Marty, P.H., Alemany, A.: Chaotic behaviour of the Rikitake dynamo with symmetric mechanical friction and azimuthal currents. Proc. R. Soc. A 454(1975), 1835-1842 (1998)

13. Hide, R.: Structural instability of the Rikitake disk dynamo. Geophys. Res. Lett. 22(9), 1057-1059 (1995)

14. Hassard, B., Kazarinoff, N., Wan, Y.: Theory and Application of Hopf Bifurcation. Cambridge University Press, Cambridge (1981)

15. Kuznetsov, Y.A.: Elements of Applied Bifurcation Theory. Springer, New York (1998)

16. Wei, Z.C., Moroz, I., Wang, Z., Sprott, J.C., Kapitaniak, T.: Dynamics at infinity, degenerate Hopf and zero-Hopf bifurcation for Kingni-Jafari system with hidden attractors. Int. J. Bifurc. Chaos 26, 1650125 (2016)

17. Bao, J.H., Yang, Q.G.: Darboux integrability of the stretch-twist-fold flow. Nonlinear Dyn. 76, 797-807 (2014)

18. Llibre, J., Zhang, X.: Darboux theory of integrability in image taking into account the multiplicity. J. Differ. Equ. 246, $541-551$ (2009) 
19. Valls, C.: Darboux integrability of a nonlinear financial system. Appl. Math. Comput. 218, 3297-3302 (2011)

20. Cao, J.L., Llibre, J., Zhang, X.: Darboux integrability and algebraic limit cycles for a class of polynomial differential systems. Sci. China Math. 57, 775-794 (2014)

21. Llibre, J., Oliveira, R., Valls, C.: On the Darboux integrability of a three-dimensional forced-damped differential system. J. Nonlinear Math. Phys. 24(4), 473-494 (2017)

22. Chavarriga, J., Grau, M.: A family of non-Darboux-integrable quadratic polynomial differential systems with algebraic solutions of arbitrarily high degree. Appl. Math. Lett. 16(6), 833-837 (2003)

23. Llibre, J., Mahdi, A., Valls, C.: Darboux integrability of the Lü system. J. Geom. Phys. 63(63), 118-128 (2013)

24. Moulin-Ollagnier, J.: Polynomial first integrals of the Lotka-Volterra system. Bull. Sci. Math. 121, 463-476 (1997)

25. Chavarriga, J., Llibre, J.: Invariant algebraic curves and rational first integrals for planar polynomial vector fields. J. Differ. Equ. 169(1), 1-16 (2001)

26. Cairó, L., Llibre, J.: Darboux integrability for 3D Lotka-Volterra systems. J. Phys. A 33, 2395-2406 (2000)

27. Llibre, J., Zhang, X.: Darboux theory of integrability in $C^{n}$ taking into account the multiplicity. J. Differ. Equ. 246(2), 541-551 (2009)

28. Christopher, C., Llibre, J., Pereira, J.V.: Multiplicity of invariant algebraic curves in polynomial vector fields. Pac. J. Math. 229, 63-117 (2007)

\section{Submit your manuscript to a SpringerOpen ${ }^{\circ}$ journal and benefit from:}

- Convenient online submission

- Rigorous peer review

- Open access: articles freely available online

- High visibility within the field

- Retaining the copyright to your article

Submit your next manuscript at $\mathbf{s p r i n g e r o p e n . c o m ~}$ 